Research Article

\title{
Principle Research on a Novel Piezoelectric 12-DOF Force/Acceleration Sensor
}

\author{
Jun Liu, ${ }^{1,2}$ Chuan-Wei Liang, ${ }^{1,2}$ Min Li, ${ }^{1,2}$ Ke Jian,, ${ }^{1,2}$ Lan Qin, ${ }^{1}$ and Jing-Cheng Liu ${ }^{1}$ \\ ${ }^{1}$ Key Laboratory of Optoelectronics Technology and Systems Ministry of Education, Chongqing University, Chongqing 400044, China \\ ${ }^{2}$ College of Optoelectronic Engineering, Chongqing University, Chongqing 400044, China
}

Correspondence should be addressed to Jun Liu; junliu@cqu.edu.cn

Received 2 July 2017; Accepted 9 October 2017; Published 31 December 2017

Academic Editor: Stefano Stassi

Copyright (C) 2017 Jun Liu et al. This is an open access article distributed under the Creative Commons Attribution License, which permits unrestricted use, distribution, and reproduction in any medium, provided the original work is properly cited.

\begin{abstract}
This study proposes a new piezoelectric 12-DOF force/acceleration sensor structure to measure forces, torques, and accelerations, during a robot's space motion. The study involves analyzing the operating principle and structural characteristics in order to obtain the model structure of a sensor. The mechanical diagram of the sensor was drawn based on the structural parameters of a piezoelectric 12-DOF force/acceleration sensor, and a numerical simulation model was established. The sensor utilizes piezoelectric quartz, of different cutting types, as the sensing and conversion element. Additionally, ANSYS was used to study the static sensitivity, crossing couplings, natural frequency, and other characteristics. The research results indicate that the piezoelectric 12-DOF force/acceleration sensor has many advantages, which include a simple structure, high integration, good linearity, and dynamic characteristics. The sensor's operating principle is accurate, and the crossing couplings correspond to linear coupling. The results of the static characteristic analysis are consistent with the structural model. The natural frequencies exceed $11 \mathrm{kHz}$, and the relative errors of output data are less than $1 \%$, with respect to the decoupling calculation.
\end{abstract}

\section{Introduction}

A 12-DOF force/acceleration sensor achieves sensing and measurement of spatial force information, three-dimensional linear acceleration, and three-dimensional angular acceleration relative to an inertia coordinate system. The system is mainly used for measuring robotic force/torques and linear/angular accelerations, loading identification, extraction of contact forces, motion control, and other aspects where it is necessary to simultaneously detect six-DOF force/torque and six-DOF linear/angular acceleration $[1,2]$. This corresponds to broad application prospects in humanoid robots, automotive, aerospace, deep-sea exploration, and other fields. Due to a complex structure and calibration difficulties, a few research institutions across the world are committed to investment, research, and development with respect to the sameness.

A multidimensional force/acceleration sensor can be classified into elastic-style and nonelastic-style sensor in the light of the existence or nonexistence of the independent elastic body [3]. An elastic-style multidimensional force/ acceleration sensor always exists two major contradictions caused by the independent elastic body between the complex degree of elastic body structure and strain gage distribution [4] as well as decoupling, high rigidity, and high sensitivity [5]. So far, scholars in this field mainly focus on elastic-style 12-DOF force/acceleration sensor. A strain-type 12-DOF force/acceleration sensor with a double cross beam structure has been presented by JR3 Inc. [6]. It involved an inertia mass, which was mounted on the cross beam and was used as a NASA space manipulator. YanShan University [7] proposed a 12-DOF force/acceleration sensor by using a double-parallel Stewart structure. The inner ring of the Stewart platform was equipped with an inertial mass for measuring 6-DOF acceleration, and the Stewart 6-DOF force sensor, in the outer ring, measured the 6-DOF force. The 6-axis force sensing and 6-axis acceleration sensing parts shared the pedestal. A measurement scheme of a 12-DOF force/acceleration sensor, in which four groups (eight) of two-axis acceleration sensors were installed in the inner ring of a cross beam 6-axis force sensor, has been reported by the Harbin Institute of Technology $[8,9]$. 
This was presented as a solution to the residual vibration, difficulty of identifying the load parameters, and contactimpact problems of a 6-axis force sensor, during spatial movement. Kröger et al. [10] established a minimal custom setup for the 12-DOF force/acceleration by using a JR3 6-DOF force sensor in combination with a set of MEMS. Specifically, three pairs of MEMS were arranged in angles of $120^{\circ}$, on a cylindrical adapter part, and every pair of the MEMS consisted of two single-axis orthogonal MEMS accelerometers.

In order to overcome the above contradictions existed in elastic-style 12-DOF force/acceleration sensor, two types of inelastic-style 12-DOF force/acceleration sensors based on piezoelectric element are presented. Piezoelectric quartz is chosen for two types of inelastic-style sensors' sensing element and conversion element and senses the measured quantities directly. Because of the nonexistence of independent elastic body, these schemes are able to overcome the above contradictions existed in elastic-style 12-DOF force/acceleration sensor. In the first project, installation base of six-axis force sensing unit and six-DOF acceleration sensing unit was shared [11]. Though that can decrease the volume, the vibration generated by 6-axis force sensing unit under alternating forces disturbed the 6 -axis acceleration sensing unit, and decoupling cannot be achieved.

To solve the first project's problem, the paper proposes the second research approach in which an acceleration shell is used for combining 6-axis acceleration sensing unit and 6-axis force sensing unit together. There is a short distance between two sensing parts, and the installation base is not shared.

This paper proposes a piezoelectric 12-DOF force/ acceleration sensor based on a study carried out on a piezoelectric 6-axis force sensor [12], and a single mass piezoelectric 6-DOF accelerometer [3, 13]. The structural and numerical models of the sensor were established based on ANSYS. Additionally, the main characteristics, including sensitivity, natural frequency, and static coupling interference, were examined. Finally, the correctness of the theory of piezoelectric 12-DOF force/acceleration was verified.

\section{Measuring Principle and Structural Model}

The 6-axis force sensing and 6-DOF acceleration sensing parts, in the piezoelectric 12-DOF force/acceleration sensor, are shown in Figures 1(a) and 1(b), respectively. The 6-axis force sensing part consists of (1) force shell, (2) pretension nut, (3) cover, (4) pretension bolt, (5) piezoelectric quartz crystal chip group unit, and (6) pedestal; the 6-DOF acceleration sensing part consists of (7) pretension bolt, (8) inertial mass, (9) piezoelectric quartz crystal chip group unit, (10) pedestal, and (11) acceleration shell. The piezoelectric quartz crystal chip groups, of the 6-axis force sensing part and the 6-axis acceleration sensing part, were sandwiched between pedestal (6) and cover (3), and pedestal (10) and inertial mass (8).

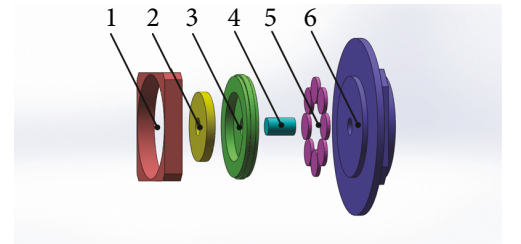

(a)

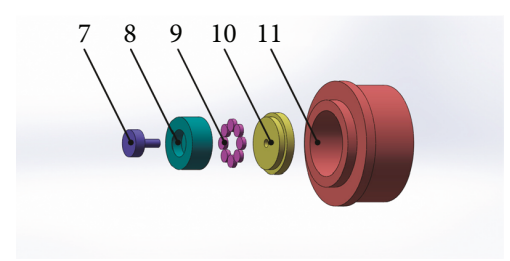

(b)

FIgURE 1: A schematic diagram of a 12-DOF force/acceleration sensor. (a) 6-axis force sensing part of a piezoelectric 12-DOF force/acceleration sensor; (b) 6-DOF acceleration sensing part of a piezoelectric 12-DOF force/acceleration sensor.

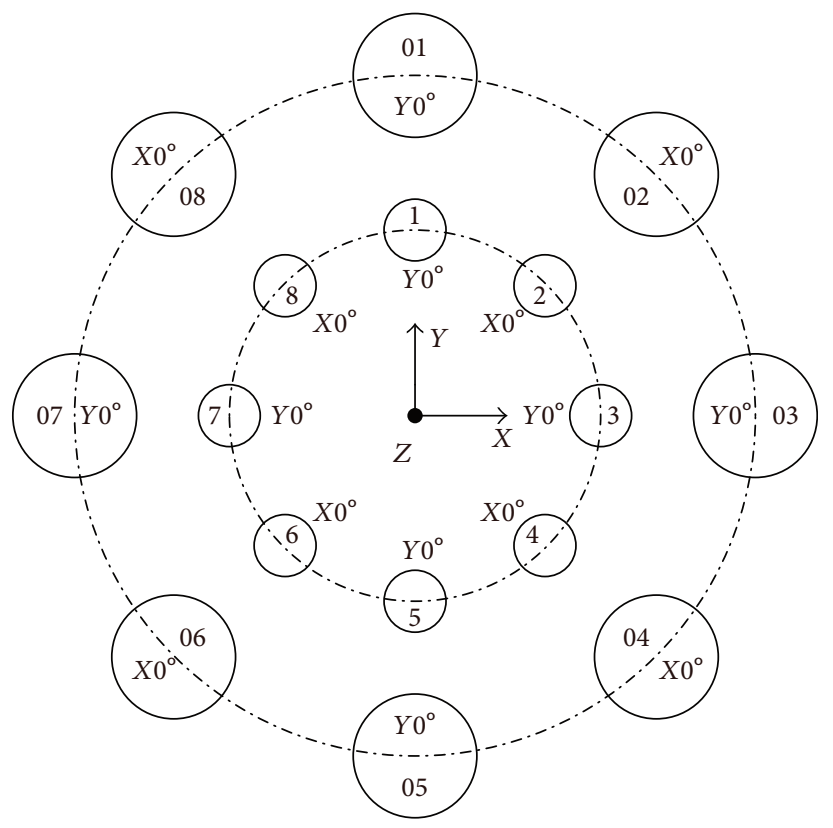

FIgURe 2: A layout diagram of piezoelectric quartz crystal chip groups.

2.1. Working Principle. The distribution of the two piezoelectric quartz crystal chip group units, in a piezoelectric 12-DOF force/acceleration sensor, is identical, as shown in Figure 2. It is assumed that the measured space forces and torques correspond to $f_{x}, f_{y}, f_{z}, m_{x}, m_{y}$, and $m_{z}$; the measured linear acceleration and angular acceleration correspond to $a_{x}, a_{y}, a_{z}, \alpha_{x}, \alpha_{y}$, and $\alpha_{z}$; the response outputs of the 6-axis acceleration sensing and 6-axis force sensing parts correspond to $A_{x}, A_{y}, A_{z}, A_{\alpha x}, A_{\alpha y}, A_{\alpha z}, F_{x}$, $F_{y}, F_{z}, M_{x}, M_{y}$, and $M_{z}$.

In the piezoelectric 12-DOF force/acceleration sensor, two piezoelectric quartz crystal chip group units were 
uniformly distributed in concentric circles. Quartz crystal chip groups $1,3,5$, and 7 correspond to $Y 0^{0}$ crystals and are responsible for the measurement of three parameters in the directions of $a_{x}, a_{y}$, and $\alpha_{z}$. Additionally, four groups of quartz crystal chip groups of $X 0^{0}$ crystals were distributed to the remaining four positions and were responsible for the measurement of three parameters in the directions of $a_{z}, \alpha_{x}$, and $\alpha_{y}$. Similarly, quartz crystal chip groups $1,3,5$, and 7 correspond to the $Y 0^{0}$ crystals in charge of the measurement of three parameters in the directions of $f_{x}, f_{y}$, and $m_{z}$. Furthermore, four groups of quartz crystal chip groups of $X 0^{0}$ crystals were distributed to the remaining four positions in charge of the measurement of three parameters in the directions of $f_{z}, m_{x}$, and $m_{y}$. Each set of piezoelectric quartz crystal chip group corresponds to a one-channel output signal. Thus, the 16-channel signal, of a piezoelectric 12-DOF force/acceleration sensor, was preprocessed and converted into a 12channel signal, and the measured parameters were obtained from the 12-channel signal by performing a decoupling matrix calculation and error correction. Equation (1) denotes the measuring principle of the mapping relationships of a piezoelectric 12-DOF force/acceleration sensor, as follows:

$$
\begin{aligned}
A_{x} & =k_{a_{x}}\left(F_{1}-F_{5}\right), \\
A_{y} & =k_{a_{y}}\left(F_{3}-F_{7}\right), \\
A_{z} & =k_{a_{z}}\left(F_{2}+F_{4}+F_{6}+F_{8}\right), \\
A_{\alpha_{x}} & =k_{\alpha_{x}}\left[\left(F_{2}+F_{8}\right)-\left(F_{4}+F_{6}\right)\right], \\
A_{\alpha_{y}} & =k_{\alpha_{y}}\left[\left(F_{6}+F_{8}\right)-\left(F_{2}+F_{4}\right)\right], \\
A_{\alpha_{z}} & =k_{\alpha_{z}}\left(F_{1}+F_{3}+F_{5}+F_{7}\right), \\
F_{x} & =k_{f_{x}}\left(F_{01}-F_{05}\right), \\
F_{y} & =k_{f_{y}}\left(F_{03}-F_{07}\right), \\
F_{z} & =k_{f_{z}}\left(F_{02}+F_{04}+F_{06}+F_{08}\right), \\
M_{x} & =k_{m_{x}}\left[\left(F_{02}+F_{08}\right)-\left(F_{04}+F_{06}\right)\right] r_{1}, \\
M_{y} & =k_{m_{y}}\left[\left(F_{06}+F_{08}\right)-\left(F_{02}+F_{04}\right)\right] r_{1}, \\
M_{z} & =k_{m_{z}}\left(F_{01}+F_{03}+F_{05}+F_{07}\right) R_{1} .
\end{aligned}
$$

In (1), $F_{i}$ represents the inertial force sensed by the piezoelectric quartz crystal chip groups in the 6-axis acceleration sensing part, and $F_{0 i}$ represents the contact force sensed by the piezoelectric quartz crystal chip groups in the 6-axis force sensing part. The subscript numbers represent the serial number of quartz crystal chip groups, and the subscript letters represent the spatial axes of the piezoelectric 12-DOF force/acceleration sensor. The force and the acceleration transfer coefficients $\mathrm{K}$ were determined by the level of processing technology, parameters, and forms of sensor structure. They represent the ratio of the actual force, or acceleration, measured by piezoelectric quartz crystal chip groups for external force, or

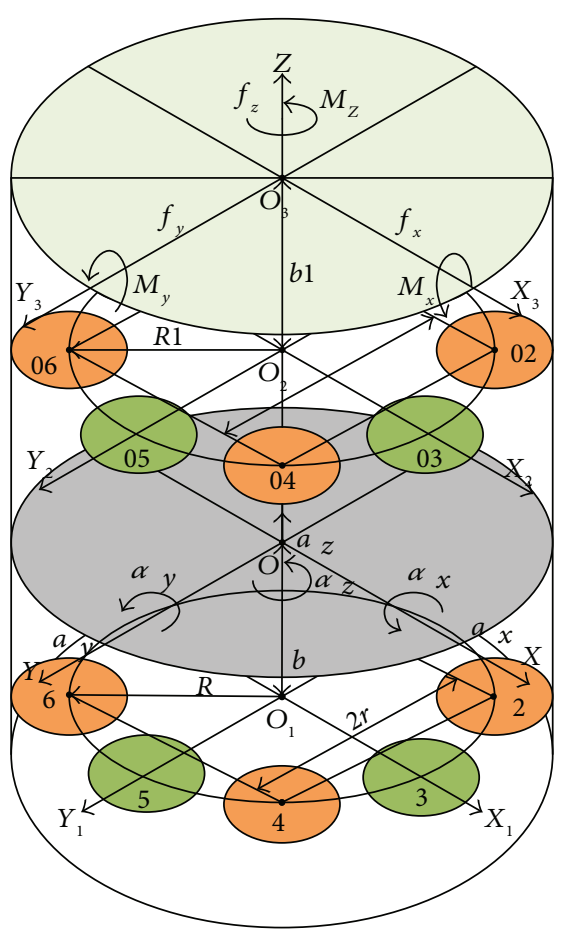

FIgURe 3: A structural diagram of the piezoelectric 12-DOF force/ acceleration sensor.

acceleration, when the external force/torque or inertial force/torque is distributed by a pretension nut, cover, and other nonconverting elements.

2.2. Structural Model. The following assumptions were adopted in order to simplify the analysis: the rigidities of the quartz crystal chip groups, as well as the same sensitivity and symmetrical uniform layout, are identical. Both the inertial mass and cover correspond to rigid bodies and possess equal rigidity in all directions. Additionally, they possess identical sensitivity and are distributed uniformly. The distributions of $f_{z}, m_{x}$, and $m_{y}$ obey the lever principle of quartz crystal chip groups, and $f_{x}, f_{y}$, and $m_{z}$ are distributed equally. Similarly, the distributions of $a_{z}, \alpha_{x}$, and $\alpha_{y}$ conform to the lever principle on quartz crystal chip groups, and $a_{x}, a_{y}$, and $\alpha_{z}$ are distributed equally.

Figure 3 shows the structure diagram of the piezoelectric 12-DOF force/acceleration sensor. In the figure, Oxyz denotes the installation coordinate system of the 12-DOF force/acceleration sensor; $O_{1} x_{1} y_{1} z_{1}$ denotes the installation coordinate system of the acceleration quartz crystal chip groups, in which the distribution radius corresponds to $R, r=R / \sqrt{2}$, and the distance between the inertial mass centroid and the surface of the quartz crystal chip groups corresponding to $b$. The coordinate system of the installed quartz crystal chip groups, of the 6-axis force sensing part, corresponds to $\mathrm{O}_{2} x_{2} y_{2} z_{2}$, and the coordinate system of the point of the measured force application of the 6-axis force sensing part correspond to $\mathrm{O}_{3} x_{3} y_{3} z_{3}$. The distribution radius of the force quartz crystal chip groups corresponds 
to $R 1$, such that $R_{1}=\sqrt{2} \cdot r_{1}$. The distance between the point of force application and the surface of quartz crystal chip groups corresponds to $b 1$.

$$
\begin{aligned}
F_{1}= & \frac{a_{x}}{8}+\frac{\alpha_{z}}{8 R}+\frac{\alpha_{y}}{R}, \\
F_{2}= & \frac{a_{z}}{8}+\frac{\left[-a_{y} b-a_{x} b+\alpha_{x}-\alpha_{y}\right]}{3 r}, \\
F_{3}= & \frac{a_{y}}{8}+\frac{\alpha_{z}}{8 R}-\frac{\alpha_{x}}{R}, \\
F_{4}= & \frac{a_{z}}{8}+\frac{\left[a_{y} b-a_{x} b-\alpha_{x}-\alpha_{y}\right]}{3 r}, \\
F_{5}= & \frac{-a_{x}}{8}+\frac{\alpha_{z}}{8 R}-\frac{\alpha_{y}}{R}, \\
F_{6}= & \frac{a_{z}}{8}+\frac{\left[a_{y} b+a_{x} b-\alpha_{x}+\alpha_{y}\right]}{3 r}, \\
F_{7}= & \frac{-a_{y}}{8}+\frac{\alpha_{z}}{8 R}+\frac{\alpha_{x}}{R}, \\
F_{8}= & \frac{a_{z}}{8}+\frac{\left[-a_{y} b+a_{x} b+\alpha_{x}+\alpha_{y}\right]}{3 r}, \\
F_{01}= & \frac{f_{x}}{8}+\frac{m_{z}}{8 R_{1}}+\frac{m a_{x}}{8}+\frac{J_{z} \alpha_{z}}{8 R_{1}}+\frac{J_{y} \alpha_{y}}{R_{1}}, \\
F_{02}= & \frac{f_{z}}{8}+\frac{m a_{z}}{8}+\frac{\left[-f_{y} b_{1} / 8-f_{x} b_{1} / 8+m_{x} / 6-m_{y} / 6\right]}{r_{1}} \\
& +\frac{\left[-m a_{y} b_{1}-m_{x} b_{1}+J_{x} \alpha_{x}-J_{y} \alpha_{y}\right]}{3 r_{1}}
\end{aligned}
$$$$
F_{03}=\frac{f_{y}}{8}+\frac{m_{z}}{8 R_{1}}+\frac{m a_{y}}{8}+\frac{J_{z} \alpha_{z}}{8 R_{1}}-\frac{J_{x} \alpha_{x}}{R_{1}},
$$$$
F_{04}=\frac{f_{z}}{8}+\frac{m a_{z}}{8}+\frac{\left[f_{y} b_{1} / 8-f_{x} b_{1} / 8-m_{x} / 6-m_{y} / 6\right]}{r_{1}}
$$$$
+\frac{\left[m a_{y} b_{1}-m a_{x} b_{1}-J_{x} \alpha_{x}-J_{y} \alpha_{y}\right]}{3 r_{1}},
$$$$
F_{05}=\frac{-f_{x}}{8}+\frac{m_{z}}{8 R_{1}}-\frac{m a_{x}}{8}+\frac{J_{z} \alpha_{z}}{8 R_{1}}-\frac{J_{y} \alpha_{y}}{R_{1}},
$$$$
F_{06}=\frac{f_{z}}{8}+\frac{m a_{z}}{8}+\frac{\left[f_{y} b_{1} / 8+f_{x} b_{1} / 8-m_{x} / 6+m_{y} / 6\right]}{r_{1}}
$$$$
+\frac{\left[m a_{y} b_{1}+m a_{x} b_{1}-J_{x} \alpha_{x}+J_{y} \alpha_{y}\right]}{3 r_{1}}
$$$$
F_{07}=\frac{-f_{y}}{8}+\frac{m_{z}}{8 R_{1}}-\frac{m a_{y}}{8}+\frac{J_{z} \alpha_{z}}{8 R_{1}}+\frac{J_{x} \alpha_{x}}{R_{1}},
$$$$
F_{08}=\frac{f_{z}}{8}+\frac{m a_{z}}{8}+\frac{\left[-f_{y} b_{1} / 8+f_{x} b_{1} / 8+m_{x} / 6+m_{y} / 6\right]}{r_{1}}
$$$$
+\frac{\left[-m a_{y} b_{1}+m a_{x} b_{1}+J_{x} \alpha_{x}+J_{y} \alpha_{y}\right]}{3 r_{1}},
$$

$$
\begin{aligned}
& A_{x}=\left(\frac{a_{x}}{4}+\frac{2 \alpha_{y}}{R}\right) k_{a_{x}}, \\
& A_{y}=\left(\frac{a_{y}}{4}+\frac{2 \alpha_{x}}{R}\right) k_{a_{y}} \text {, } \\
& A_{z}=\left(\frac{a_{z}}{2}\right) k_{a_{z}}, \\
& A_{\alpha_{x}}=\left(\frac{4 \alpha_{x}}{3 r}-\frac{4 a_{y} b}{3 r}\right) k_{\alpha_{x}}, \\
& A_{\alpha_{y}}=\left(\frac{4 \alpha_{y}}{3 r}+\frac{4 a_{x} b}{3 r}\right) k_{\alpha_{y}}, \\
& A_{\alpha_{z}}=\left(\frac{\alpha_{z}}{2 R}\right) k_{\alpha_{z}}, \\
& F_{x}=\left(\frac{f_{x}}{4}+\frac{m a_{x}}{4}+\frac{2 J_{y} \alpha_{y}}{R_{1}}\right) k_{f_{x}}, \\
& F_{y}=\left(\frac{f_{y}}{4}+\frac{m a_{y}}{4}+\frac{2 J_{x} \alpha_{x}}{R_{1}}\right) k_{f_{y}} \text {, } \\
& F_{z}=\left(\frac{f_{z}}{2}+\frac{m a_{z}}{2}\right) k_{f_{z}}, \\
& M_{x}=\left(\frac{2 m_{x}}{3}-\frac{f_{y} b}{2}+\frac{4 J_{x} \alpha_{x}}{3}-\frac{4 m a_{y} b}{3}\right) k_{m_{x}} \text {, } \\
& M_{y}=\left(\frac{2 m_{y}}{3}+\frac{f_{x} b}{2}+\frac{4 J_{y} \alpha_{y}}{3}+\frac{4 m a_{x} b}{3}\right) k_{m_{y}} \text {, } \\
& M_{z}=\left(\frac{m_{z}}{2}+\frac{J_{z} \alpha_{z}}{2}\right) k_{m_{z}} .
\end{aligned}
$$

The 6-DOF acceleration sensing part was in the form of an equivalent inertial force, in order to measure the acceleration in the piezoelectric 12-DOF force/acceleration sensor. The inertial coupling in the inertial field doubly impacts the response outputs of the 6-axis force sensing part, with respect to force sand acceleration loads. The response output of each quartz crystal chip group, in the sensor for the spatial 6-axis forces and accelerations, is shown in (2), in which $m$ denotes the cover's mass, and $J_{x}, J_{y}$, and $J_{z}$ denote the rotary inertia of the cover, with respect to the $X_{-}, Y_{-}$, and $Z$-axes, respectively.

In (3), the input-output structure model relationship, of the piezoelectric 12-DOF force/acceleration sensor, was deduced based on (1) and (2). From (3), the following conclusions were drawn: with respect to the 6-axis acceleration sensing part, mutual linear couplings always exist between $a_{y}$ and $\alpha_{x}$, as well as between $a_{x}$ and $\alpha_{y}$, while linear couplings do not exist between $a_{z}$ and $\alpha_{z}$. This is due to the influence of its own spatial structure and the inertial couplings of the 6axis force sensing part in the acceleration field. In the 6-axis force sensing part, linear couplings are generated in the directions of $a_{x}, \alpha_{y}, F_{x}$, and $M_{y}$. The directions of $a_{z}$ and $F_{z}$ together with the directions of $\alpha_{z}$ and $M_{z}$ are all the same with respect to the directions of $a_{y}, \alpha_{x}, M_{x}$, and $F_{y}$, although a linear coupling is not present between $f_{z}$ and $m_{z}$. This type of linear coupling can be eliminated by employing a mathematical method. 
TABLE 1: Main structural parameters of the sensor.

\begin{tabular}{|c|c|c|c|c|c|}
\hline Sensing part & Thickness $(\mathrm{mm})$ & Outer diameter $(\mathrm{mm})$ & Material & Elasticity modulus (GPa) & Density $\left(\mathrm{kg} / \mathrm{m}^{3}\right)$ \\
\hline F-cover & 7 & 59 & 1Cr18Ni9Ti & 210 & 7900 \\
\hline F-pretension bolt & 16 & 10 & 1Cr18Ni9Ti & 210 & 7900 \\
\hline F- mounting boss & 3 & 57 & 1Cr18Ni9Ti & 210 & 7900 \\
\hline F-mounting pedestal & 4 & 80 & 1Cr18Ni9Ti & 210 & 7900 \\
\hline F-piezoelectric element & 2 & 15 & $\mathrm{SiO}_{2}$ & 80 & 2650 \\
\hline A-inertial mass & 11 & 25 & 1Cr18Ni9Ti & 210 & 7900 \\
\hline A-boss & 3 & 23 & 1Cr18Ni9Ti & 210 & 7900 \\
\hline A-pedestal & 4 & 29 & 1Cr18Ni9Ti & 210 & 7900 \\
\hline A-piezoelectric element & 2 & 6 & $\mathrm{SiO}_{2}$ & 80 & 2650 \\
\hline
\end{tabular}

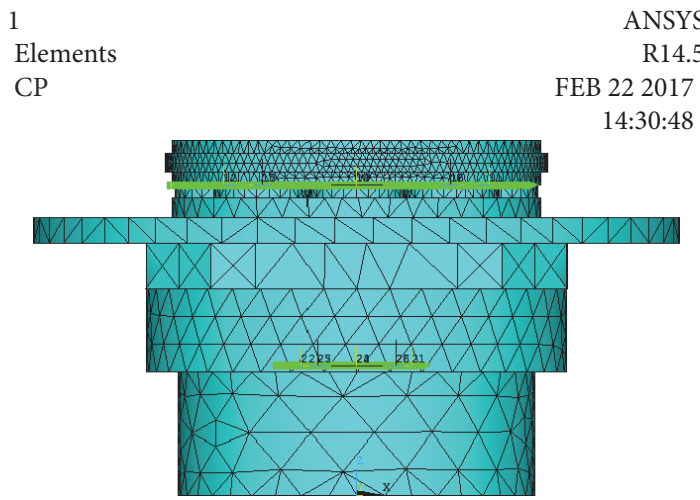

FIGURE 4: ANSYS mesh graph of the piezoelectric 12-DOF force/ acceleration sensor.

\section{Numerical Simulation Method}

In order to verify the effectiveness of the piezoelectric 12DOF force and acceleration sensor's sensing principle, an accurate, practical, and efficient numerical analysis method was presented as the preferred means of research. The static and dynamic characteristic simulation methods of the 12DOF force/acceleration sensor were created based on ANSYS, because this software possesses a powerful analysis capability with respect to the coupling of a piezoelectricity field. Table 1 provides the main structural parameters of the sensor, where $F$ represents the force sensing component and $A$ represents the acceleration sensing component.

3.1. FEM Modeling. Modeling process: based on the actual structure of a 12-DOF force/acceleration sensor, the physical structure model was first established with SolidWorks, PROE, and other CAD software. Subsequently, the physical structure model was imported into ANSYS, through the ANSYS software interface. With respect to the sensor's material property setup, the coupling unit SOLID98 was selected as the element type of the quartz crystal, while the other component material corresponded to stainless steel with the element type corresponding to SOLID186, $E X=2 \times 10^{11} \mathrm{~Pa}$ and $\mu=0.3$. A combination of manually controlling the number of equal divisions and free meshing was adopted as the meshing approach, with respect to the previously mentioned components. A load application included installation constraints, which exerted forces and accelerations. Based on the installation condition of the 12-DOF force and acceleration sensor, all the DOF, of the sensormounting pedestal's installation surface, were set to 0 . Based on the actual application mode of the sensor, 6-axis forces were applied to the top surface of the cover, and 6-axis accelerations were applied directly to the entire 12-DOF force/ acceleration sensor, in a global fashion. In order to simplify the exertion of preloads in practice, the contact states were specifically set to a perpetually bound state among the bottom surface of the cover, the piezoelectric quartz crystal chip groups of the 6-axis force sensing part, and the installation surface of the force piezoelectric quartz crystal chip groups. Similarly, the contact states were specifically set to a perpetually bound state among the bottom surface of the inertial mass, piezoelectric quartz crystal chip groups of the 6-axis acceleration sensing part, and the installation surface of the acceleration piezoelectric quartz crystal chip groups. Figure 3 shows the ANSYS mesh graph of the piezoelectric 12-DOF force and acceleration sensor.

\section{Numerical Simulation Experiment}

4.1. Analysis of Static Characteristics. After the static simulation experiment was performed, the input-output curves of force and charge, in the directions of $F_{x}, F_{y}, F_{z}, M_{x}, M_{y}$, and $M_{z}$, were derived as shown in Figure 4. Additionally, the input-output curves of acceleration and charge, in the directions of $A_{x}, A_{y}, A_{z}, \alpha_{x}, \alpha_{y}$, and $\alpha_{z}$, are shown in Figure 5. Furthermore, 192 sensor output data were received under the influence of a 12-DOF force and acceleration loads. Four $6 \times 6$ matrices, namely, $C_{F F}, C_{A A}, C_{F A}$, and $C_{A F}$, are obtained by numerical simulation experiment, as shown in (4), (5), (6), and (7). Equation (4) denotes a voltage sensitivity matrix of a 6-axis force sensing unit under 6-axis forces. Equation (6) denotes a voltage sensitivity matrix of a 6-axis acceleration sensing unit under 6-axis forces. Equation (5) denotes a voltage sensitivity matrix of a 6 -axis acceleration sensing unit under 6-axis accelerations. And (7) denotes a voltage sensitivity matrix of a 6 -axis force sensing unit under 6-axis accelerations. The calculation of the 12DOF force and acceleration loads is achieved by using the above four equations. 


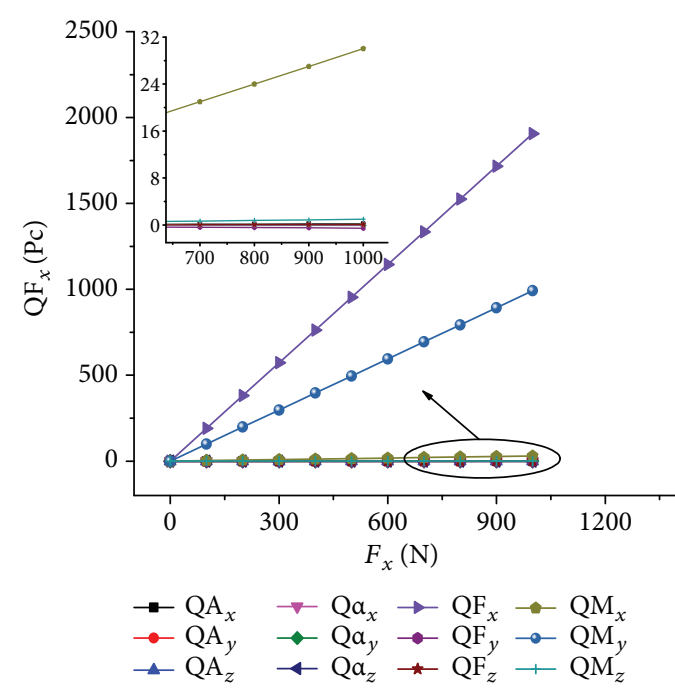

(a)

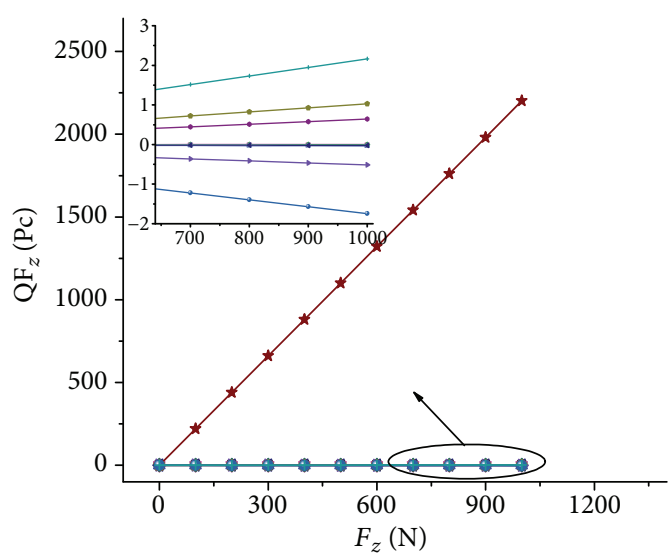

$\rightarrow \mathrm{QA}_{x} \rightarrow \mathrm{Qd}_{x} \rightarrow \mathrm{QF}_{x} \rightarrow \mathrm{QM}_{x}$

$\bullet \mathrm{QA}_{y} \rightarrow \mathrm{Qa}_{y} \rightarrow \mathrm{QF}_{y} \rightarrow \mathrm{QM}_{y}$

$\neg \mathrm{QA}_{z} \leftarrow \mathrm{Qa}_{z} \star \mathrm{QF}_{z}+\mathrm{QM}_{z}$

(c)

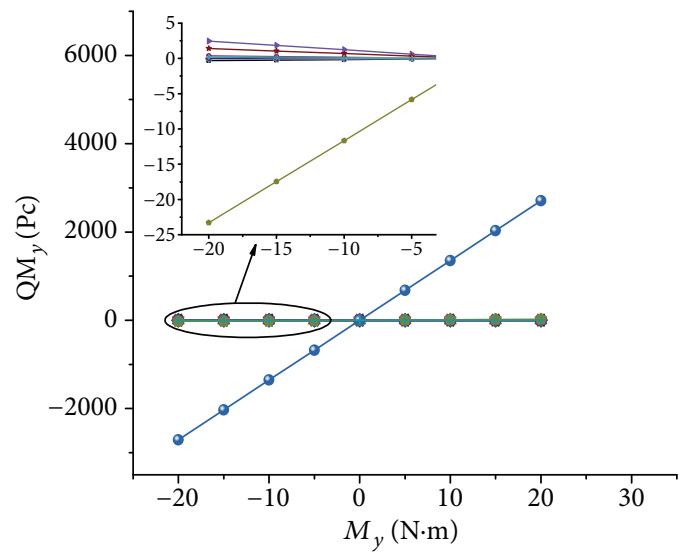

$\because \mathrm{QA}_{x} \rightarrow \mathrm{Qd}_{x} \rightarrow \mathrm{QF}_{x} \rightarrow \mathrm{QM}_{x}$
$\because \mathrm{QA}_{y} \multimap \mathrm{Qd}_{y} \rightarrow \mathrm{QF}_{y} \rightarrow \mathrm{QM}_{y}$
$\because \mathrm{QA}_{z} \multimap \mathrm{Qd}_{z} \nleftarrow \mathrm{QF}_{z} \rightarrow \mathrm{QM}_{z}$

(e)

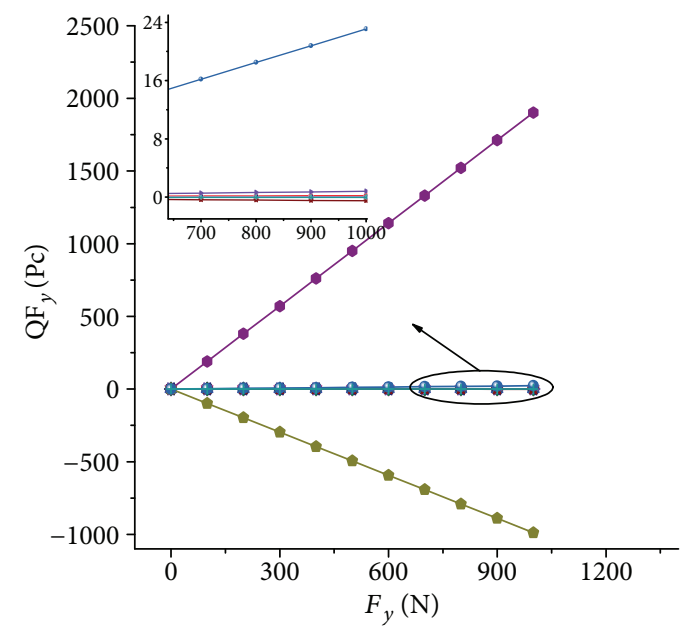

$\rightarrow \mathrm{QA}_{x} \rightarrow \mathrm{Qa}_{x} \rightarrow \mathrm{QF}_{x} \rightarrow-\mathrm{QM}_{x}$

$\rightarrow \mathrm{QA}_{y} \rightarrow \mathrm{Qd}_{y} \rightarrow \mathrm{QF}_{y} \rightarrow \mathrm{QM}_{y}$

$\neg \mathrm{QA}_{z} \leftarrow \mathrm{Qa}_{z} \star \mathrm{QF}_{z} \rightarrow \mathrm{QM}_{z}$

(b)

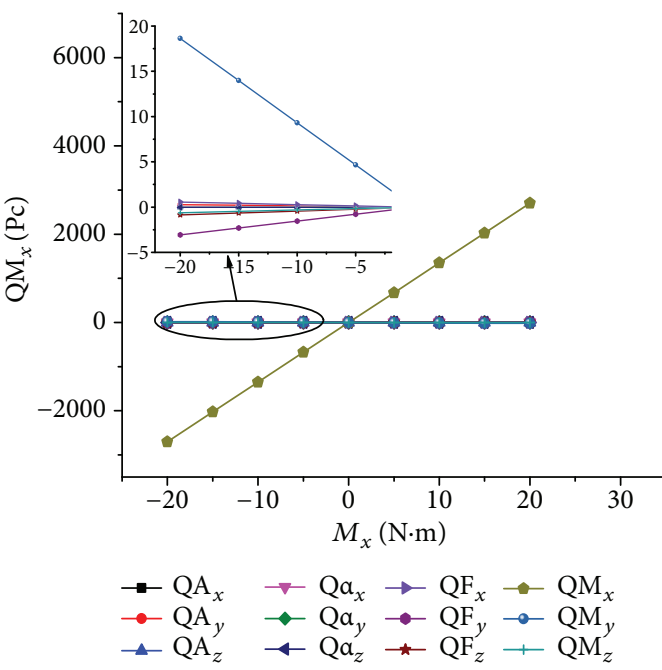

(d)

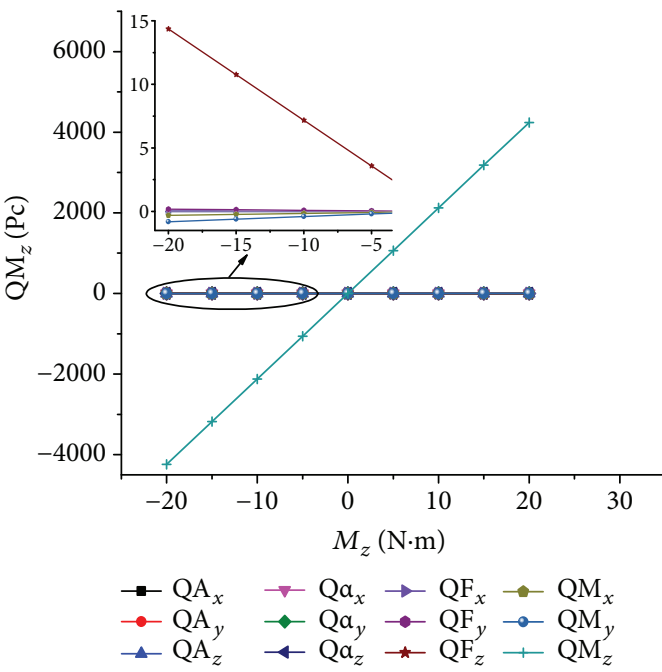

(f)

Figure 5: Input-output curves of force and the charge of the 6-axis force sensing part. (a) $F_{x}$, (b) $F_{y}$, (c) $F_{z}$, (d) $M_{x}$, (e) $M_{y}$, and (f) $M_{z}$. 


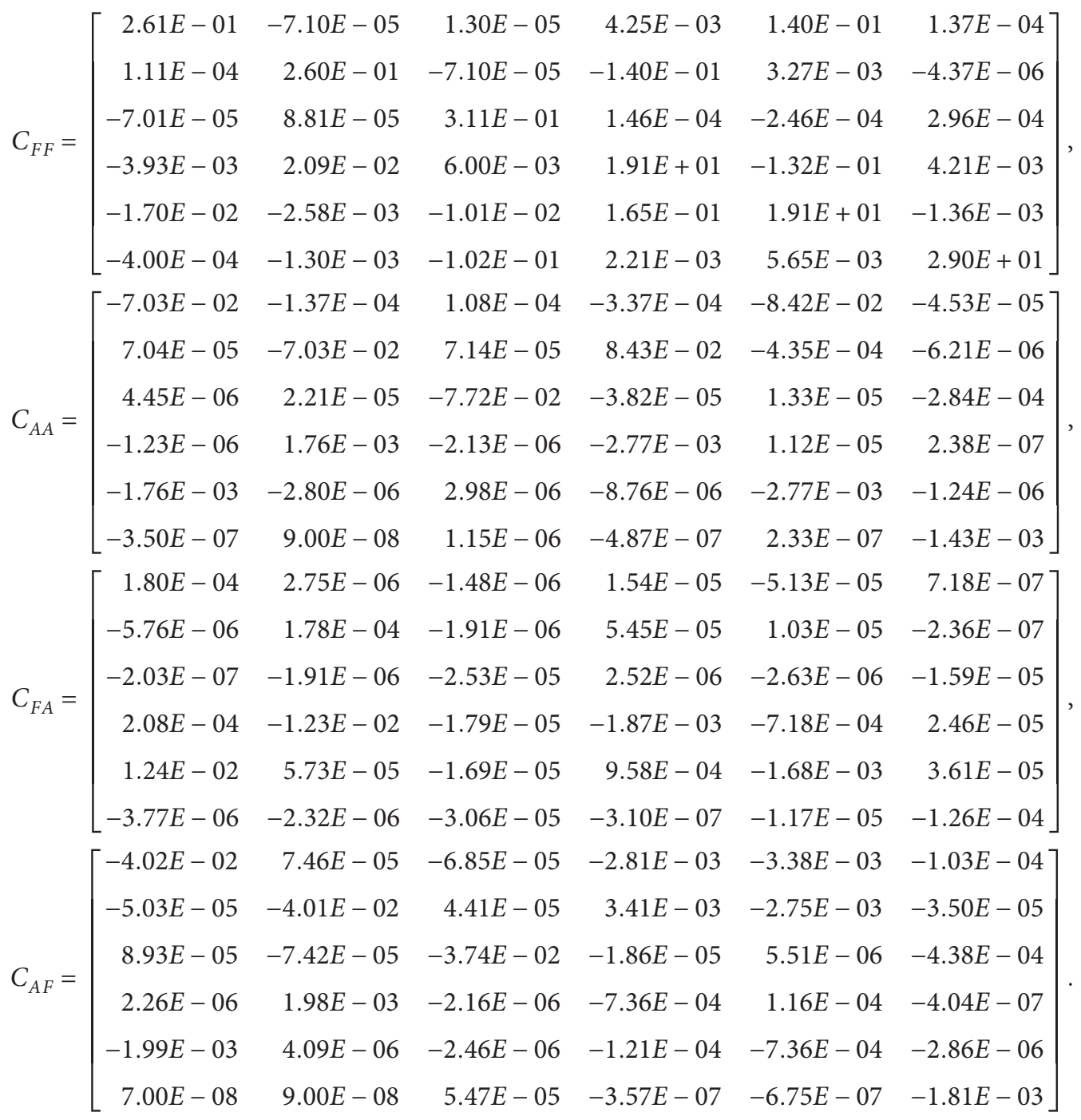

TABLE 2: Crossing coupling simulation results of static sensitivity in 6-axis force directions under an external force.

\begin{tabular}{|c|c|c|c|c|c|c|}
\hline \multirow{2}{*}{ Load category } & \multicolumn{6}{|c|}{ Crossing coupling } \\
\hline & $F_{x}$ & $F_{y}$ & $F_{z}$ & $M_{x}$ & $M_{y}$ & $M_{z}$ \\
\hline$F_{x}$ & 1 & $-0.0272 \%$ & $0.0048 \%$ & $1.5753 \%$ & $52.0007 \%$ & $0.0527 \%$ \\
\hline$F_{y}$ & $0.0427 \%$ & 1 & $-0.0264 \%$ & $-51.9716 \%$ & $1.2153 \%$ & $-0.0017 \%$ \\
\hline$F_{z}$ & $-0.0233 \%$ & $0.0293 \%$ & 1 & $0.0469 \%$ & $-0.0790 \%$ & $0.0983 \%$ \\
\hline$M_{x}$ & $-0.0213 \%$ & $0.1134 \%$ & $0.0314 \%$ & 1 & $-0.6897 \%$ & $0.0228 \%$ \\
\hline$M_{y}$ & $-0.0918 \%$ & $-0.0140 \%$ & $-0.0528 \%$ & $0.8605 \%$ & 1 & $-0.0074 \%$ \\
\hline$M_{z}$ & $-0.0014 \%$ & $-0.0045 \%$ & $-0.3384 \%$ & $0.0074 \%$ & $0.0188 \%$ & 1 \\
\hline
\end{tabular}

According to Figure 5, (4) and (7), the sensitivity characteristics and the conclusion of the crossing coupling, of the 6 -axis force sensing unit, are available as shown in Tables 2 and 3. The 6-axis charge sensitivities, of the 6-axis force sensing unit, correspond to $1.91 \mathrm{pC} / \mathrm{N}, 1.91 \mathrm{pC} / \mathrm{N}, 2.20 \mathrm{pC} / \mathrm{N}$,
$135.09 \mathrm{pC} /(\mathrm{N} \cdot \mathrm{m}), \quad 135.29 \mathrm{pC} /(\mathrm{N} \cdot \mathrm{m})$, and $212.0 \mathrm{pC} /(\mathrm{N} \cdot \mathrm{m})$. The crossing coupling and linear coupling only appear between $f_{x}$ and $m_{y}$, as well as between $f_{y}$ and $m_{x}$. The above conclusions are consistent with the 12-axis static sensitivity characteristic analysis, which was obtained from the 
TABLE 3: Crossing coupling simulation results of static sensitivity in 6-axis acceleration directions under an external force.

\begin{tabular}{|c|c|c|c|c|c|c|}
\hline \multirow{2}{*}{ Load category } & \multicolumn{6}{|c|}{ Crossing coupling } \\
\hline & $A_{x}$ & $A_{y}$ & $A_{z}$ & $\alpha_{x}$ & $\alpha_{y}$ & $\alpha_{z}$ \\
\hline$F$ & $0.0111 \%$ & $0.0002 \%$ & $-0.0001 \%$ & $0.0009 \%$ & $-0.0030 \%$ & 0 \\
\hline$F_{y}$ & $-0.0004 \%$ & $0.0110 \%$ & $-0.0001 \%$ & $0.0032 \%$ & $0.0006 \%$ & 0 \\
\hline$F_{z}$ & 0 & $-0.0001 \%$ & $-0.0013 \%$ & $0.0001 \%$ & $-0.0001 \%$ & $-0.0008 \%$ \\
\hline$M_{x}$ & $0.0002 \%$ & $-0.0106 \%$ & 0 & $-0.0016 \%$ & $-0.0006 \%$ & 0 \\
\hline$M_{y}$ & $0.0107 \%$ & 0 & 0 & $0.0008 \%$ & $-0.0014 \%$ & 0 \\
\hline$M_{z}$ & 0 & 0 & 0 & 0 & 0 & $-0.0001 \%$ \\
\hline
\end{tabular}

structure model of a 12-DOF force/acceleration sensor in the force field.

The sensitivity characteristic and conclusion of the crossing coupling of the 6-axis acceleration sensing unit were obtained on the basis of Figure 6, (5) and (6), as shown in Tables 4 and 5. The 6-axis charge sensitivities of the 6-axis acceleration sensing unit correspond to $-0.0807 \mathrm{pC} / \mathrm{g}, \quad-0.0807 \mathrm{pC} / \mathrm{g}, \quad-0.0856 \mathrm{pC} / \mathrm{g}, \quad-0.0031 \mathrm{pC} /$ $\mathrm{rad} \cdot \mathrm{s}^{-2},-0.0031 \mathrm{pC} / \mathrm{rad} \cdot \mathrm{s}^{-2}$, and $-0.0017 \mathrm{pC} / \mathrm{rad} \cdot \mathrm{s}^{-2}$. With respect to the acceleration field, the load in the $A_{x}$ direction generated coupling in the directions of $\alpha_{y}, F_{x}$, and $M_{y}$; the load in the $A_{y}$ direction generated coupling in the directions of $\alpha_{x}, F_{y}$, and $M_{x}$; the load in the $A_{z}$ direction generated coupling in the direction of $F_{z}$; the load in the $\alpha_{x}$ direction generated coupling in the directions of $A_{y}, M_{x}$, and $F_{y}$; the load in the $\alpha_{y}$ direction generated coupling in the directions of $A_{x}$, $M_{y}$, and $F_{x}$; and the load in the $\alpha_{z}$ direction generated coupling in the direction of $M_{z}$. This conclusion conformed completely to the analysis of static sensitivity and coupling characteristics, of the 12-DOF force/acceleration sensor, in the structural model.

It is necessary to discuss the results and the manner in which the results can be interpreted in the perspective of previous studies and the working hypotheses. The findings and their implications should be discussed in the broadest context possible. Future research directions may also be highlighted.

4.2. Dynamic Characteristic Simulation Experiment. With respect to the dynamic characteristics of a piezoelectric 12DOF force/acceleration sensor, this study mainly focuses on the natural frequency. The ANSYS modal and harmonic analysis method are used to estimate the sensor's natural frequency. The first 12 natural frequencies and vibrational modes, of the piezoelectric 12-DOF force and acceleration sensor, are presented in Table 6 and Figure 7.

As shown in Table 6 and Figure 7, the vibration direction, in which the same direction of force and generated acceleration loads is identical in the modal analysis, and the vibration modes and natural frequency of force and acceleration loads could not be effectively identified. Thus, the harmonic analysis method was adopted in order to further analyze the natural frequency of the sensor. Figure 8 represents the amplitude-frequency and phase-frequency characteristic curves of the 6-axis forces/torques. Figure 9 represents the amplitude-frequency and phase-frequency characteristic curves of 6-axis linear and angular accelerations.
The resonance principle and phenomenon of beat vibration caused significant changes to occur, with respect to the amplitude and phase of the 12-DOF force and acceleration sensor, at the point of resonance or beat vibration, under an excitation signal in the harmonic analysis. According to the changes in amplitude and phase, in the amplitudefrequency and phase-frequency characteristic curves of the 6 -axis forces and accelerations, the fundamental frequencies of the piezoelectric 12-DOF force/acceleration sensor were obtained in the direction of the 6-axis forces/torques and 6-axis linear/angular accelerations, that is, the sensor's natural frequencies.

With respect to the harmonic analysis of the 6-axis forces/torques, sinusoidal excitation was applied to the 12DOF force/acceleration sensor, with amplitudes corresponding to $1000 \mathrm{~N}$ and $100 \mathrm{~N} \cdot \mathrm{m}$, respectively, a frequency band range of $0 \mathrm{kHz}$ to $50 \mathrm{kHz}$, and 200 steps. The natural frequencies in the direction of the 6-axis forces and torques are shown in Table 7.

Similarly, sinusoidal excitation, with amplitudes corresponding to $490 \mathrm{~m} / \mathrm{s}^{2}$ and $1000 \mathrm{rad} / \mathrm{s}^{2}$ and a frequency band of $0 \mathrm{kHz}$ to $50 \mathrm{kHz}$ and 200 steps, was exerted on the 12 DOF force/acceleration sensor, in the harmonic analysis of the 6-axis linear/angular accelerations. The natural frequencies in the direction of the 6-axis acceleration were obtained as shown in Figure 9 by combining the peak value trends of amplitude-frequency and phase-frequency characteristic curves, in all directions, as shown in Table 8.

\section{Decoupling Method}

Figure 10 shows the strain nephogram of the piezoelectric 12DOF force/acceleration sensor under combined loading. The piezoelectric 12-DOF force/acceleration sensor was under a contact force field and an acceleration field, in a practical application. Thus, 6-axis linear/angular accelerations enabled a 6-axis force sensing part in order to generate the response outputs of the inertial forces/torques, and the 6-axis forces/ torques produced couplings in the direction of the 6-axis accelerations. Hence, the response outputs of the 6-axis force sensing unit and 6-axis acceleration sensing unit superpose and form the static crossing couplings.

The decoupling analysis of the piezoelectric 12-DOF force/acceleration sensor under combined loading is summarized in the following six steps: first-time decoupling of acceleration, first-time decoupling of force, second-time decoupling of acceleration, second-time decoupling of force, 


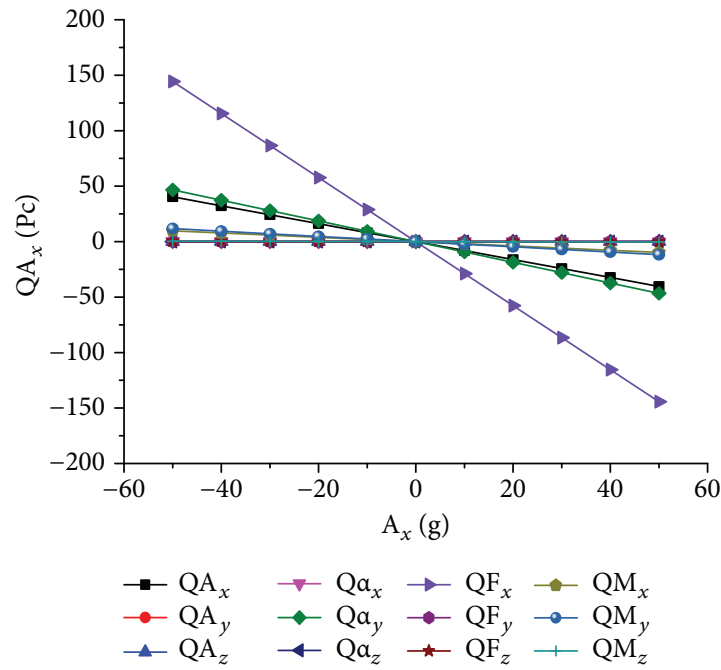

(a)

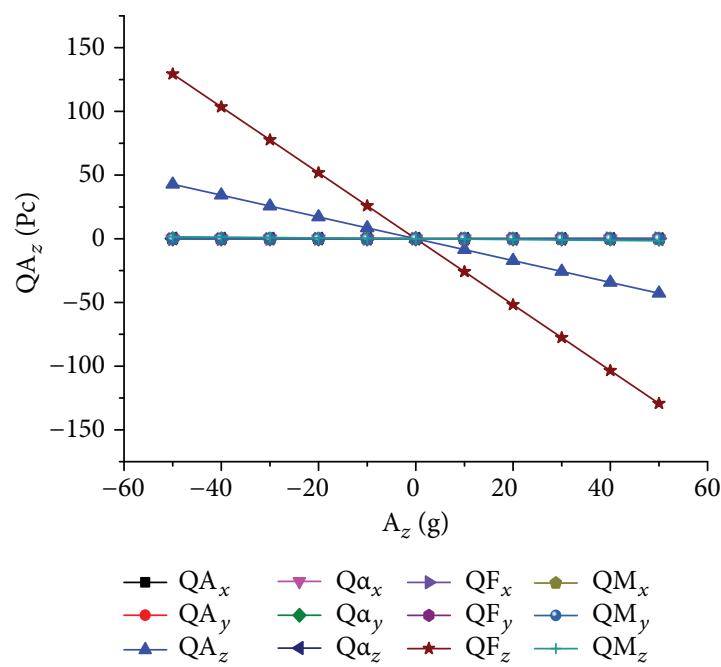

(c)

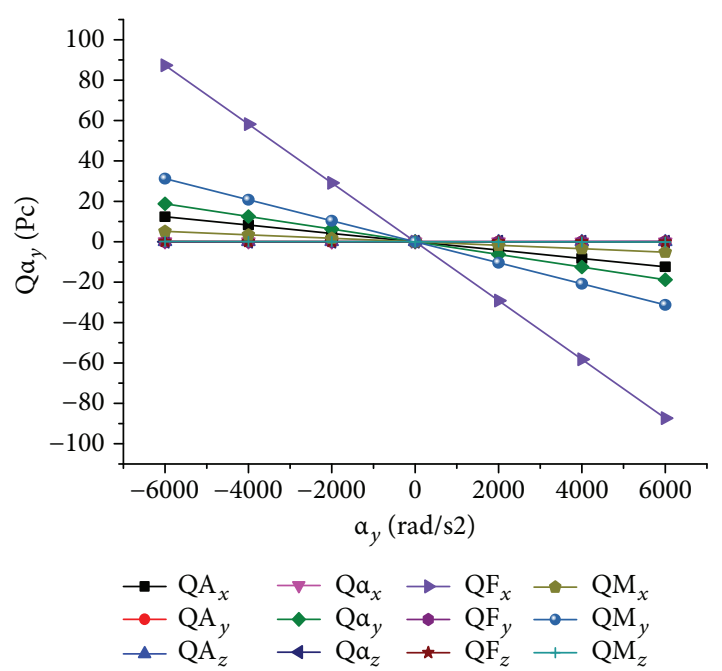

(e)

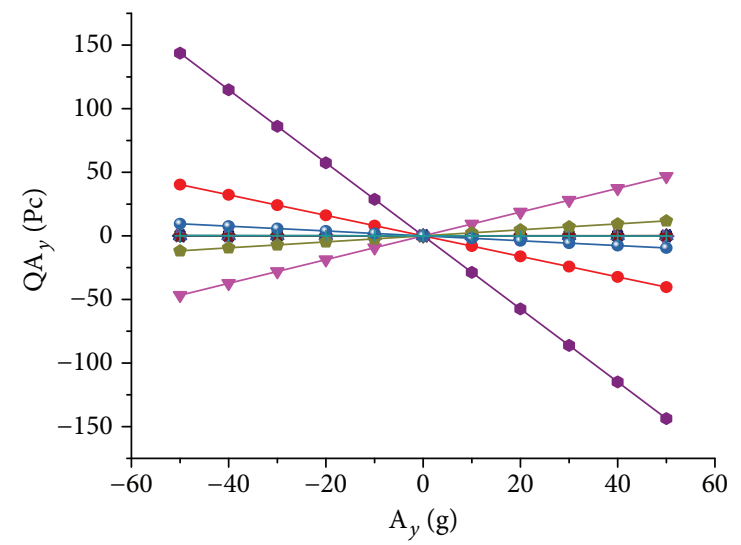

$\rightarrow \mathrm{QA}_{x} \rightarrow \mathrm{Qa}_{x} \rightarrow \mathrm{QF}_{x} \rightarrow \mathrm{QM}_{x}$

$\bullet \mathrm{QA}_{y} \rightarrow \mathrm{Qa}_{y} \rightarrow \mathrm{QF}_{y} \rightarrow \mathrm{QM}_{y}$

$\leftarrow \mathrm{QA}_{z} \leftarrow \mathrm{Qa}_{z} \rightarrow \mathrm{QF}_{z} \rightarrow \mathrm{QM}_{z}$

(b)

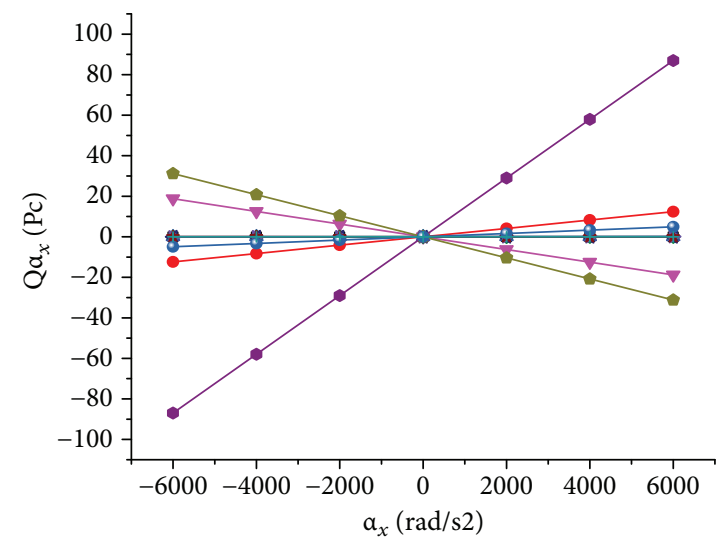

$\rightarrow \mathrm{QA}_{x} \rightarrow \mathrm{Qd}_{x} \rightarrow \mathrm{QF}_{x} \rightarrow \mathrm{QM}_{x}$

$\rightarrow \mathrm{QA}_{y} \rightarrow \mathrm{Qa}_{y} \rightarrow \mathrm{QF}_{y} \rightarrow \mathrm{QM}_{y}$

$\dashv \mathrm{QA}_{z} \longleftarrow \mathrm{Qa}_{z}$ « $\mathrm{QF}_{z} \rightarrow \mathrm{QM}_{z}^{y}$

(d)

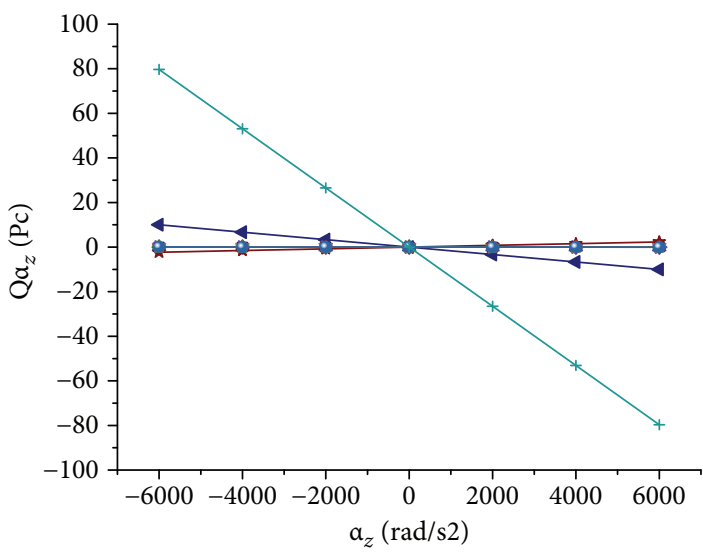

$\because \mathrm{QA}_{x} \rightarrow \mathrm{Qd}_{x} \rightarrow \mathrm{QF}_{x} \rightarrow \mathrm{QM}_{x}$
$\rightarrow \mathrm{QA}_{y} \rightarrow \mathrm{Qd}_{y} \rightarrow \mathrm{QF}_{y} \rightarrow \mathrm{QM}_{y}$
$\rightarrow \mathrm{QA}_{z} \rightarrow \mathrm{Qd}_{z} \rightarrow \mathrm{QF}_{z} \rightarrow \mathrm{QM}_{z}$

(f)

FIGURE 6: Input-output curves of acceleration and charge of the 6-axis acceleration sensing part. (a) $A_{x}$, (b) $A_{y}$, (c) $A_{z}$, (d) $\alpha_{x}$, (e) $\alpha_{y}$, and (f) $\alpha_{z}$. 
TABLE 4: Crossing coupling simulation results of static sensitivity in 6-axis acceleration directions under external acceleration.

\begin{tabular}{|c|c|c|c|c|c|c|}
\hline \multirow{2}{*}{ Load category } & \multicolumn{6}{|c|}{ Crossing coupling } \\
\hline & $A_{x}$ & $A_{y}$ & $A_{z}$ & $\alpha_{x}$ & $\alpha_{y}$ & $\alpha_{z}$ \\
\hline$A_{x}$ & 1 & $0.1942 \%$ & $-0.1485 \%$ & $0.4624 \%$ & $115.6828 \%$ & $0.0644 \%$ \\
\hline$A_{y}$ & $-0.1001 \%$ & 1 & $-0.0981 \%$ & $-115.7825 \%$ & $0.5971 \%$ & $0.0088 \%$ \\
\hline$A_{z}$ & $-0.0060 \%$ & $-0.0296 \%$ & 1 & $0.0494 \%$ & $-0.0172 \%$ & $0.3814 \%$ \\
\hline$\alpha_{x}$ & $0.0459 \%$ & $-65.8458 \%$ & $0.0768 \%$ & 1 & $-0.4050 \%$ & $-0.0089 \%$ \\
\hline$\alpha_{y}$ & $65.9159 \%$ & $0.1047 \%$ & $-0.1076 \%$ & $0.3162 \%$ & 1 & $0.0464 \%$ \\
\hline$\alpha_{z}$ & $0.0245 \%$ & $-0.0063 \%$ & $-0.0775 \%$ & $0.0329 \%$ & $-0.0158 \%$ & 1 \\
\hline
\end{tabular}

TABLE 5: Crossing coupling simulation results of static sensitivity in 6-axis force directions under external acceleration.

\begin{tabular}{|c|c|c|c|c|c|c|}
\hline \multirow{2}{*}{ Load category } & \multicolumn{6}{|c|}{ Crossing coupling } \\
\hline & $F_{x}$ & $F_{y}$ & $F_{z}$ & $M_{x}$ & $M_{y}$ & $M_{z}$ \\
\hline$A_{x}$ & $357.5465 \%$ & $-0.6633 \%$ & $0.5882 \%$ & $24.1290 \%$ & $29.0320 \%$ & $0.9147 \%$ \\
\hline$A_{y}$ & $0.4468 \%$ & $356.0423 \%$ & $-0.3784 \%$ & $-29.2919 \%$ & $23.6266 \%$ & $0.3112 \%$ \\
\hline$A_{z}$ & $-0.7484 \%$ & $0.6213 \%$ & $302.2579 \%$ & $0.1503 \%$ & $-0.0446 \%$ & $3.6708 \%$ \\
\hline$\alpha_{x}$ & $-0.5268 \%$ & $-462.1897 \%$ & $0.4869 \%$ & $165.8323 \%$ & $-26.1765 \%$ & $0.0944 \%$ \\
\hline$\alpha_{y}$ & $464.5818 \%$ & $-0.9550 \%$ & $0.5549 \%$ & $27.3322 \%$ & $166.1228 \%$ & $0.6684 \%$ \\
\hline$\alpha_{z}$ & $-0.0306 \%$ & $-0.0394 \%$ & $-23.1154 \%$ & $0.1509 \%$ & $0.2853 \%$ & $793.7115 \%$ \\
\hline
\end{tabular}

TABLe 6: Modal analysis data.

\begin{tabular}{|c|c|c|}
\hline Rank & Natural frequency $(\mathrm{Hz})$ & Vibrational mode \\
\hline 1 & 12,970 & The linear vibration of the shell along the $x$-axis \\
\hline 2 & 12977.4 & The linear vibration of the shell along the $y$-axis \\
\hline 3 & 19661.7 & The bending vibration of the lower part of the shell along the bisector of the positive $x$-and $y$-axis \\
\hline 4 & 19676.7 & $\begin{array}{c}\text { The bending vibration of the lower part of the shell along the bisector of the positive } x \text {-axis } \\
\text { and the negative } y \text {-axis }\end{array}$ \\
\hline 5 & 21005.3 & The linear vibration of the shell along the $z$-axis \\
\hline 6 & 21412.2 & The rotational vibration of the lower part of the shell along the $z$-axis \\
\hline 7 & 27467.3 & The reverse linear vibration of the upper and lower part of the shell along the $z$-axis \\
\hline 8 & 27709.7 & The bending vibration of the shell along the $y$-axis \\
\hline 9 & 27772.3 & The bending vibration of the shell along the $x$-axis \\
\hline 10 & 28833.6 & The same rotational vibration of the upper and lower part of the shell along the $z$-axis \\
\hline 11 & 35022.5 & The reverse rotational vibration of the upper and lower part of the shell along the $z$-axis \\
\hline 12 & 35,654 & $\begin{array}{l}\text { The reverse bending vibration of the upper and lower part of the shell along the bisector of the } \\
\text { positive } x \text {-axis and the negative } y \text {-axis }\end{array}$ \\
\hline
\end{tabular}

third-time decoupling of acceleration, and third-time decoupling of force. In the decoupling analysis, the first-time decoupling matrix of acceleration $A_{1}$ and the first-time decoupling matrix of force $F_{1}$ are shown in (8) and (9), respectively, in which the matrices of $V_{F F}$ and $V_{A A}$ correspond to the output potential difference matrix of the 12-DOF force/acceleration sensor in the direction of the 6-axis forces and accelerations, respectively; $G_{A A}$ and $G_{F F}$ correspond to the inverse matrices in (5) and (4), respectively, as follows:

$$
\begin{aligned}
& A_{1}=V_{A A} \cdot G_{A A}, \\
& F_{1}=V_{F F} \cdot G_{F F} .
\end{aligned}
$$

After the first two stages, the influenced matrix $A_{F-A}$, of the 6-axis acceleration sensing part by unit force, and the influenced matrix $F_{A-F}$ of the 6-axis force sensing part by unit acceleration were deduced as shown in (10) and (11), respectively, as follows:

$$
\begin{aligned}
& A_{F-A}=C_{F A} \cdot G_{A A}, \\
& F_{A-F}=C_{A F} \cdot G_{F F} .
\end{aligned}
$$

Thus, the second-time decoupling matrix of force $F_{2}$ and the second-time decoupling matrix of acceleration 


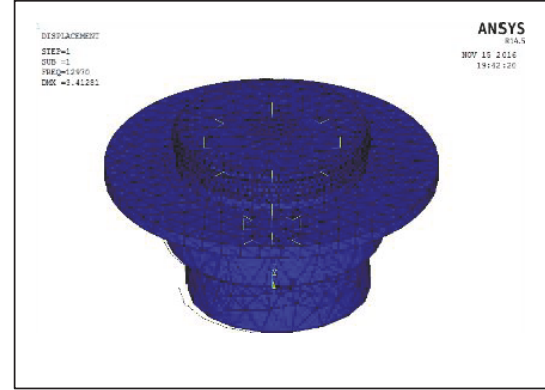

(a)

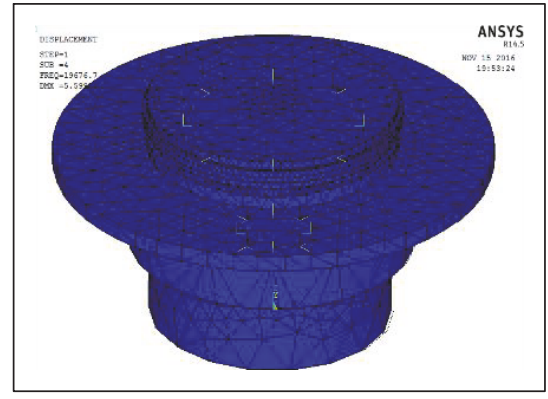

(d)

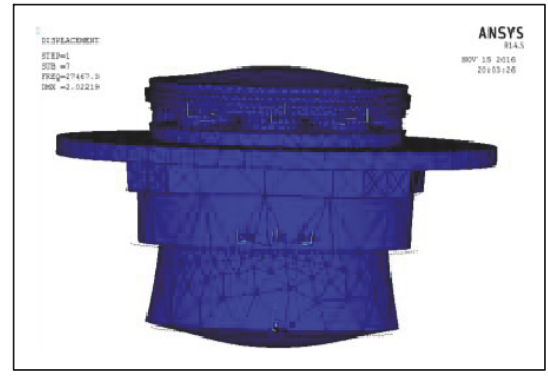

(g)

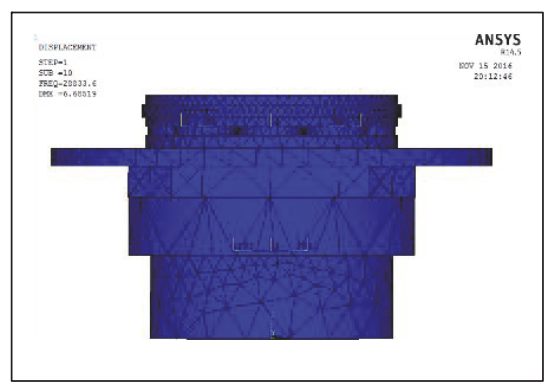

(j)

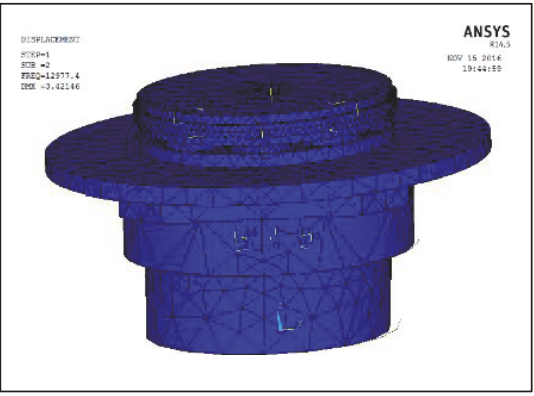

(b)

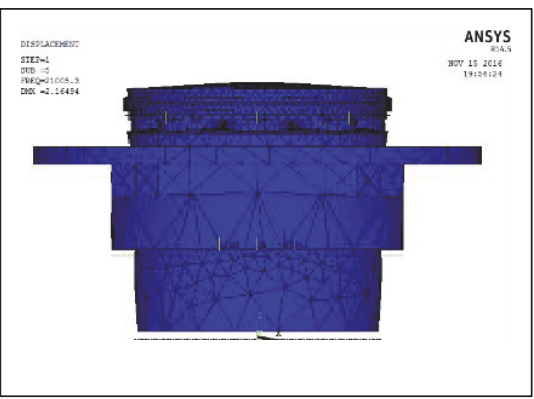

(e)

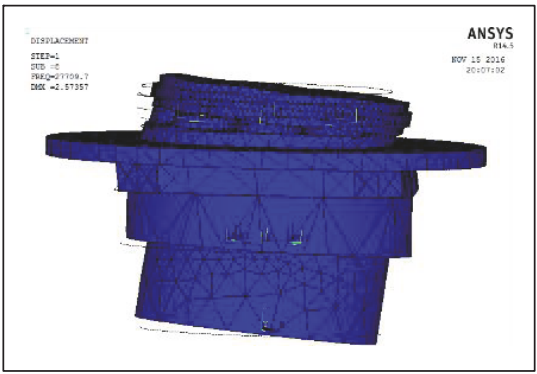

(h)

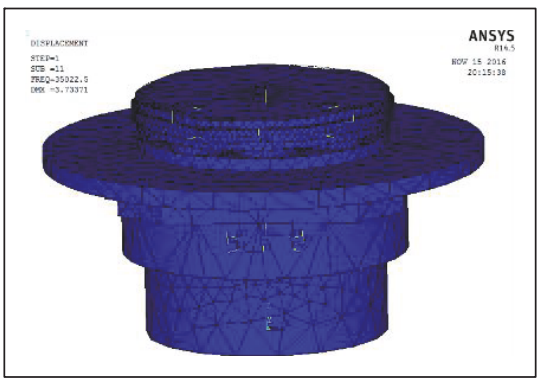

(k)

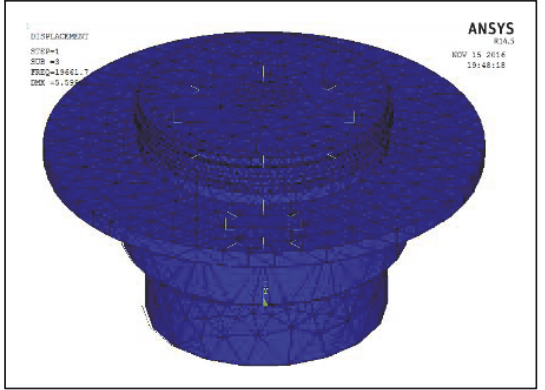

(c)

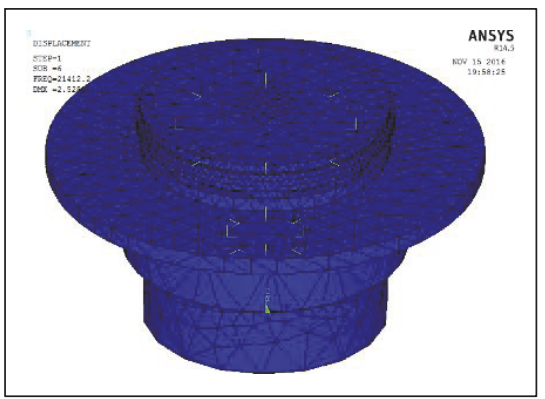

(f)

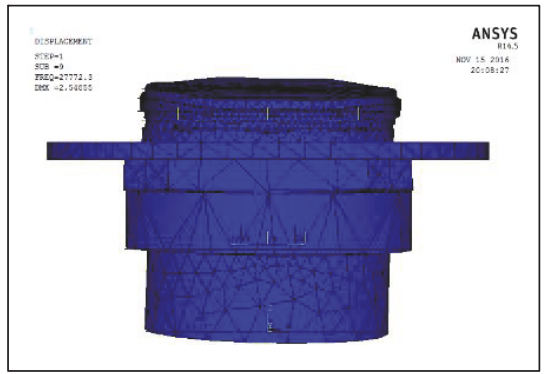

(i)

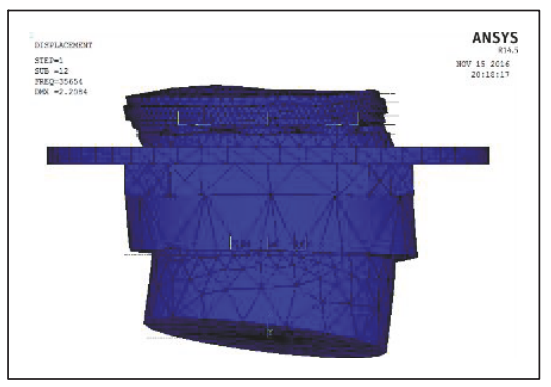

(l)

FIGURE 7: Graphs of the first 12 vibrational modes of the piezoelectric 12-DOF force/acceleration sensor. (a) First vibration mode. (b) Second vibration mode. (c) Third vibration mode. (d) Fourth vibration mode. (e) Fifth vibration mode. (f) Sixth vibration mode. (g) Seventh vibration mode. (h) Eighth vibration mode. (i) Ninth vibration mode. (j) Tenth vibration mode. (k) Eleventh vibration mode. (l) Twelfth vibration mode.

$A_{2}$ were derived as shown in (12) and (13), respectively, as follows:

$$
\begin{aligned}
& F_{2}=F_{1}-A_{1} \cdot F_{A-F}, \\
& A_{2}=A_{1}-F_{1} \cdot A_{F-A} .
\end{aligned}
$$

According to $A_{2}, F_{1}, A_{1}$, and $F_{2}$, the third-time decoupling matrix of the 6-axis force $F_{3}$ and the third-time decoupling matrix of the 6-axis acceleration $A_{3}$ were received as shown in (14) and (15), respectively, as follows:

$$
\begin{aligned}
& F_{3}=F_{1}-A_{2} \cdot F_{A-F}, \\
& A_{3}=A_{1}-F_{2} \cdot A_{F-A} .
\end{aligned}
$$




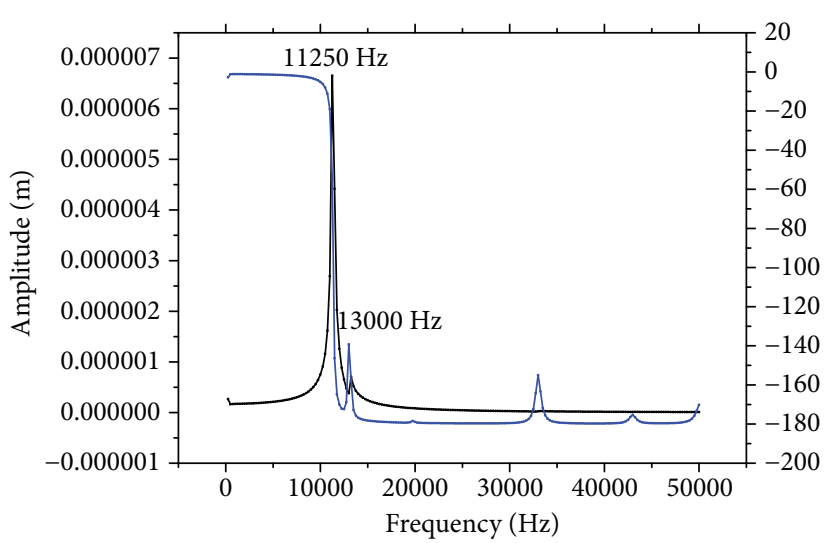

(a)

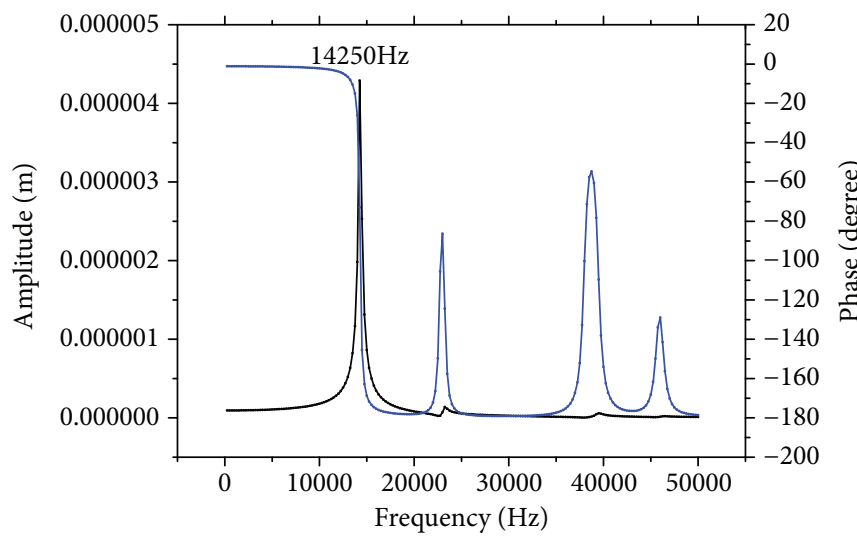

(c)

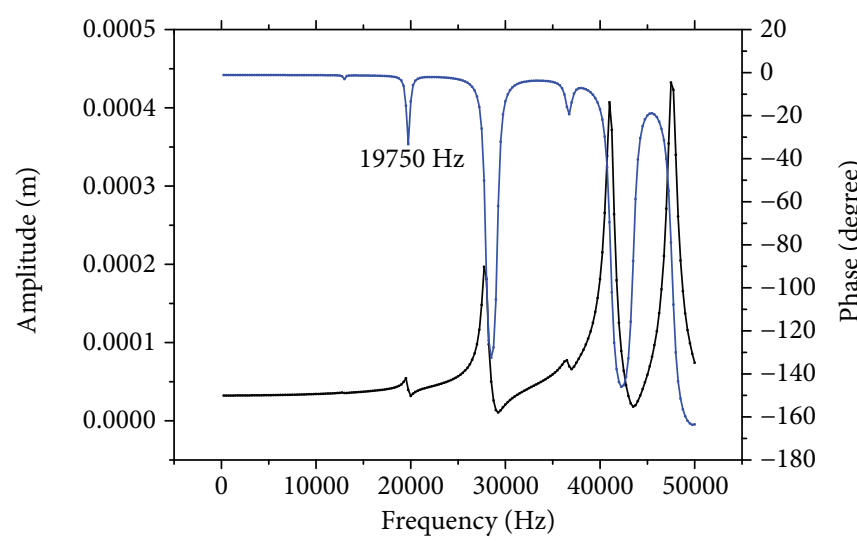

(e)

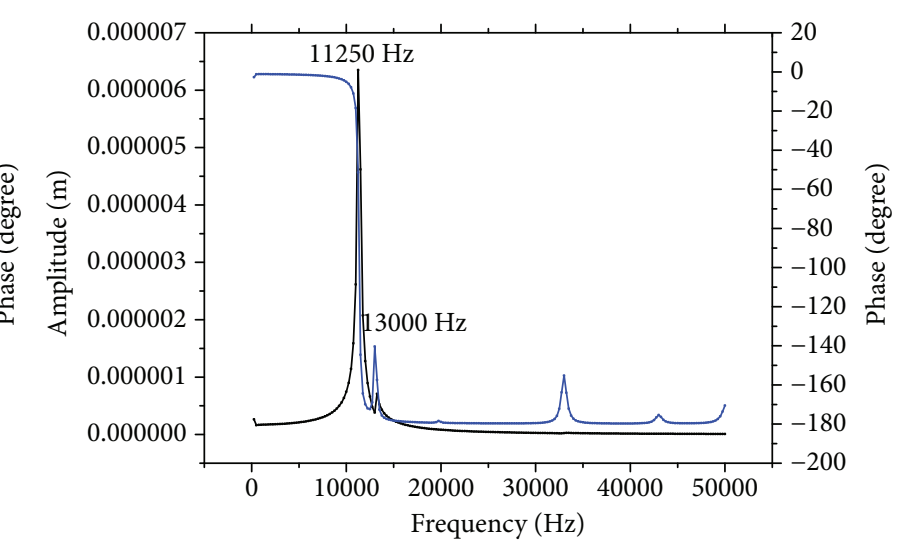

(b)

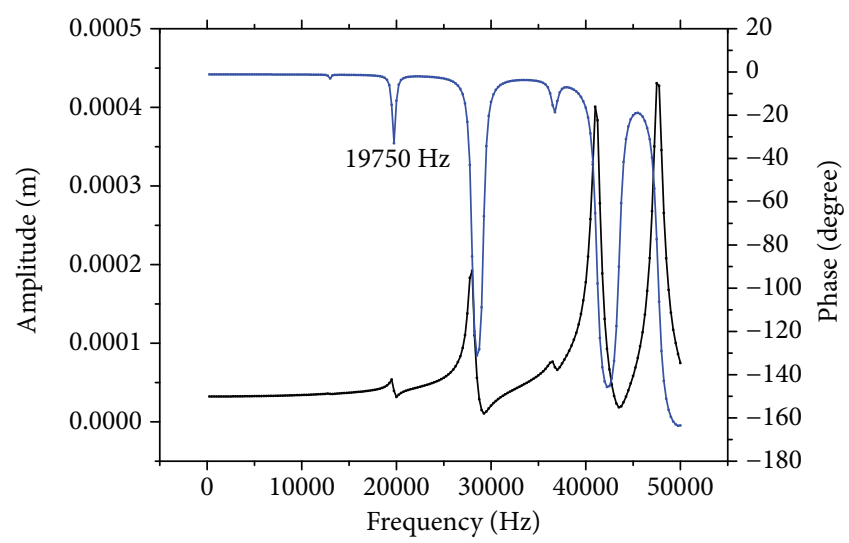

(d)

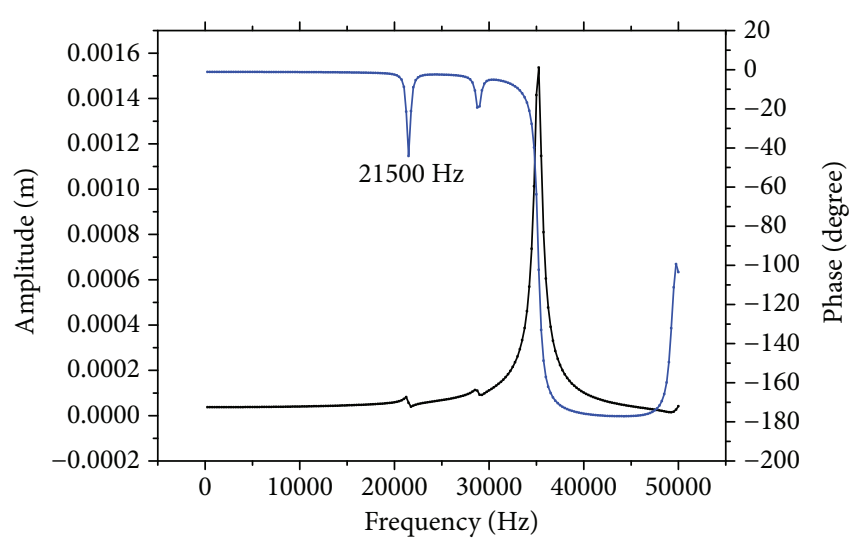

(f)

Figure 8: Amplitude-frequency and phase-frequency characteristic curves of 6-axis force/torque. (a) $F_{x}$, (b) $F_{y}$, (c) $F_{z}$, (d) $M_{x}$, (e) $M_{y}$, and (f) $M_{z}$.

After the completion of the third-time decoupling under the composite loads, the decoupling analysis results of the 6-axis force sensing part and the 6-axis acceleration sensing part were obtained by referring to $F_{1}$ and $A_{1}$, respectively, as shown in Tables 9 and 10. The data in the tables indicate that the relative error, of the 12-DOF forces and accelerations, decreased and amounted to less than $1 \%$. Thus, the effect of reducing the crossing coupling of the forces and accelerations, on the inertial field, was evident.

\section{Conclusions}

In this study, a type of piezoelectric 12-DOF force and acceleration sensor was proposed, without the crossing coupling between the 6-axis force sensing part and the 6-DOF acceleration part, in order to achieve the measurement of all spatial forces and acceleration loads. The structural model of the sensor was established, and the static sensitivity, static crossing couplings, and dynamic natural frequency were analyzed using ANSYS. A decoupling 


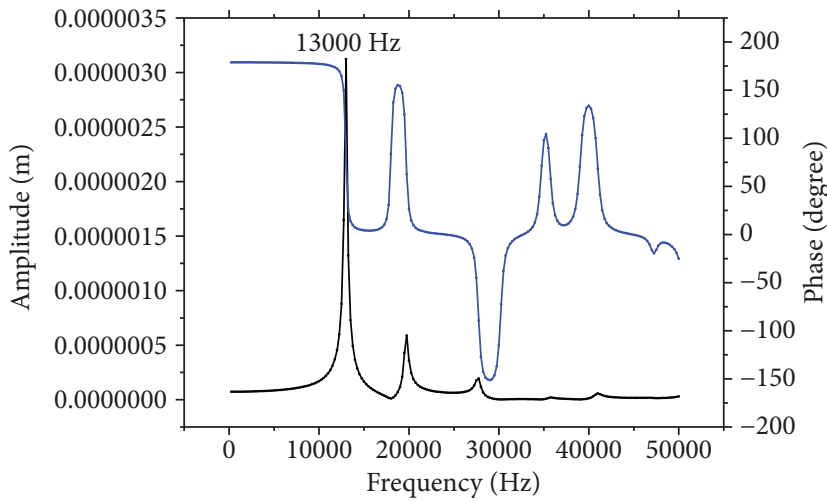

(a)

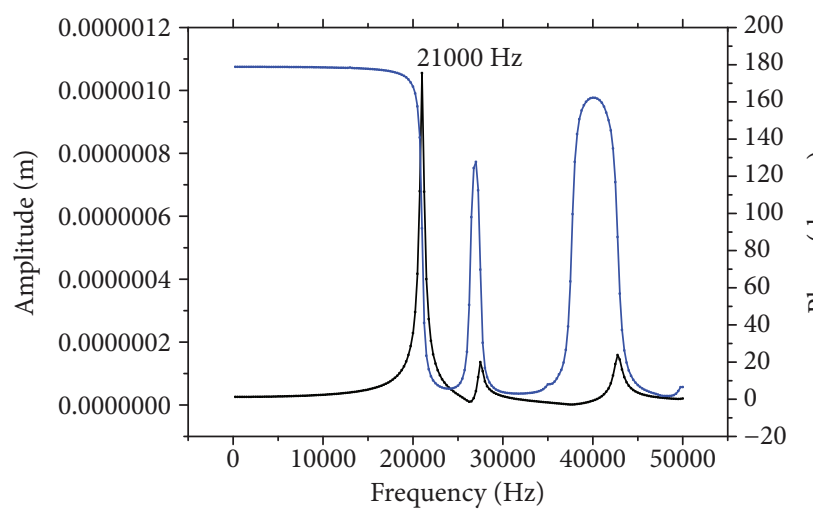

(c)

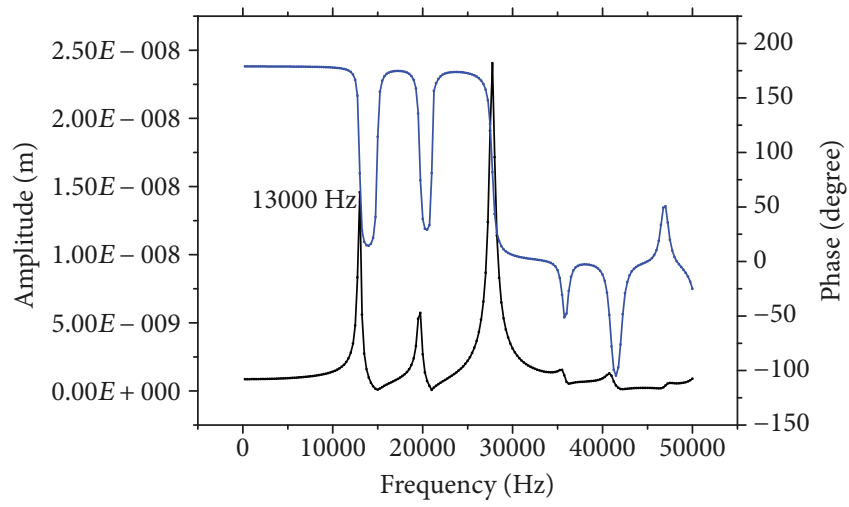

(e)

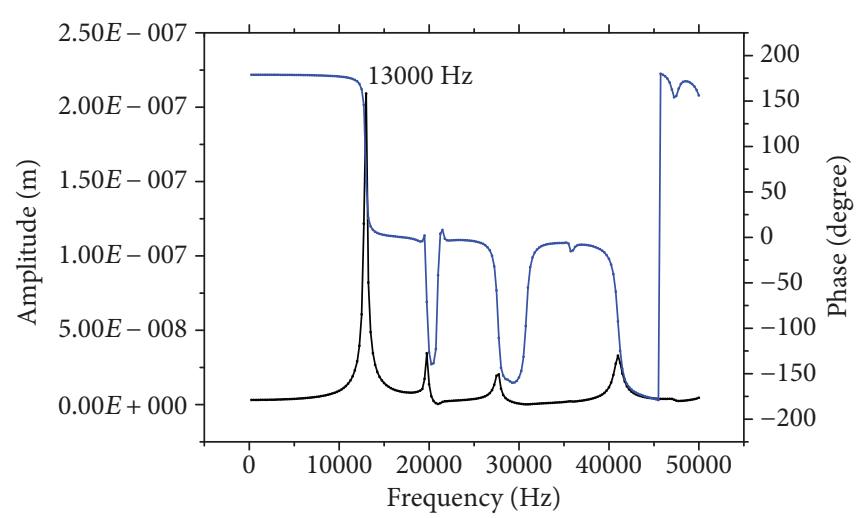

(b)

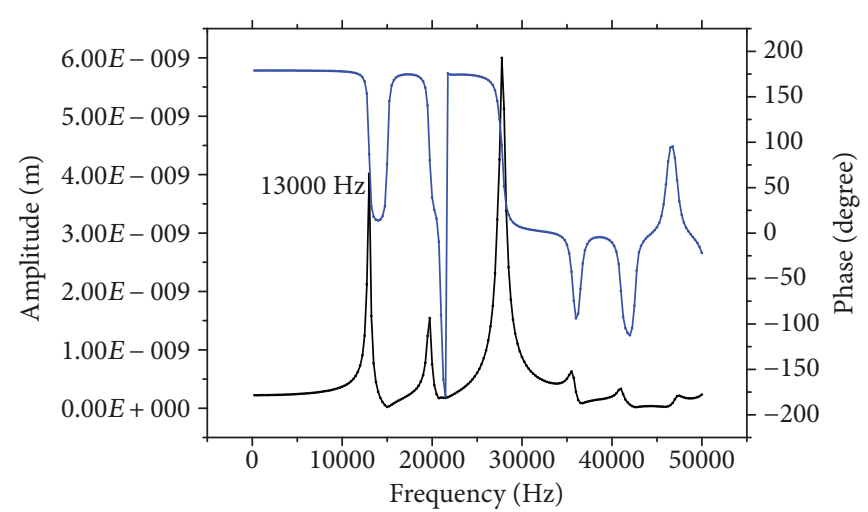

(d)

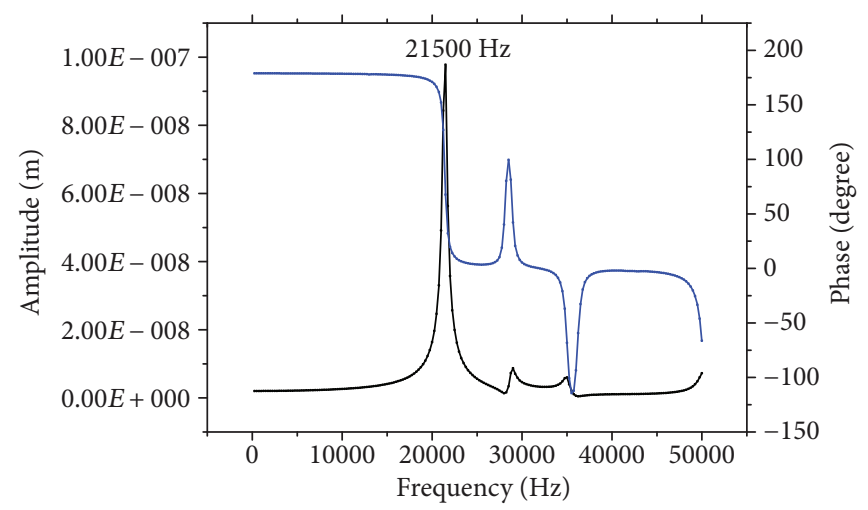

(f)

FIGURE 9: Amplitude-frequency and phase-frequency characteristic curves in the direction of 6-axis linear and angular accelerations. (a) $A_{x}$, (b) $A_{y}$, (c) $A_{z}$. (d) $\alpha_{x}$, (e) $\alpha_{y}$, and (f) $\alpha_{z}$.

TABLE 7: Natural frequency of 6-axis forces/torques.

\begin{tabular}{lccccc}
\hline$F_{x}$ & $F_{y}$ & $F_{z}$ & $M_{x}$ & $M_{y}$ & $M_{z}$ \\
\hline $11,250 \mathrm{~Hz}$ & $11,250 \mathrm{~Hz}$ & $14,250 \mathrm{~Hz}$ & $19,750 \mathrm{~Hz}$ & $19,750 \mathrm{~Hz}$ & $21,500 \mathrm{~Hz}$ \\
\hline
\end{tabular}

TABLE 8: Natural frequencies of 6-axis linear/angular accelerations.

\begin{tabular}{lcccc}
\hline$A_{x}$ & $A_{y}$ & $A_{z}$ & $\alpha_{x}$ & $\alpha_{y}$ \\
\hline $13,000 \mathrm{~Hz}$ & $13,000 \mathrm{~Hz}$ & $21,000 \mathrm{~Hz}$ & $13,000 \mathrm{~Hz}$ & $13,000 \mathrm{~Hz}$ \\
\hline
\end{tabular}


1

Nodal solution

ANSYS

STEP $=8$

R14.5

$\mathrm{SUB}=5$

NOV 152016

TIME $=8$

17:05:47

VOLT (AVG)

$\mathrm{DMX}=0.402 E-06$

$\mathrm{SMN}=-90.3111$

SMX $=101.902$
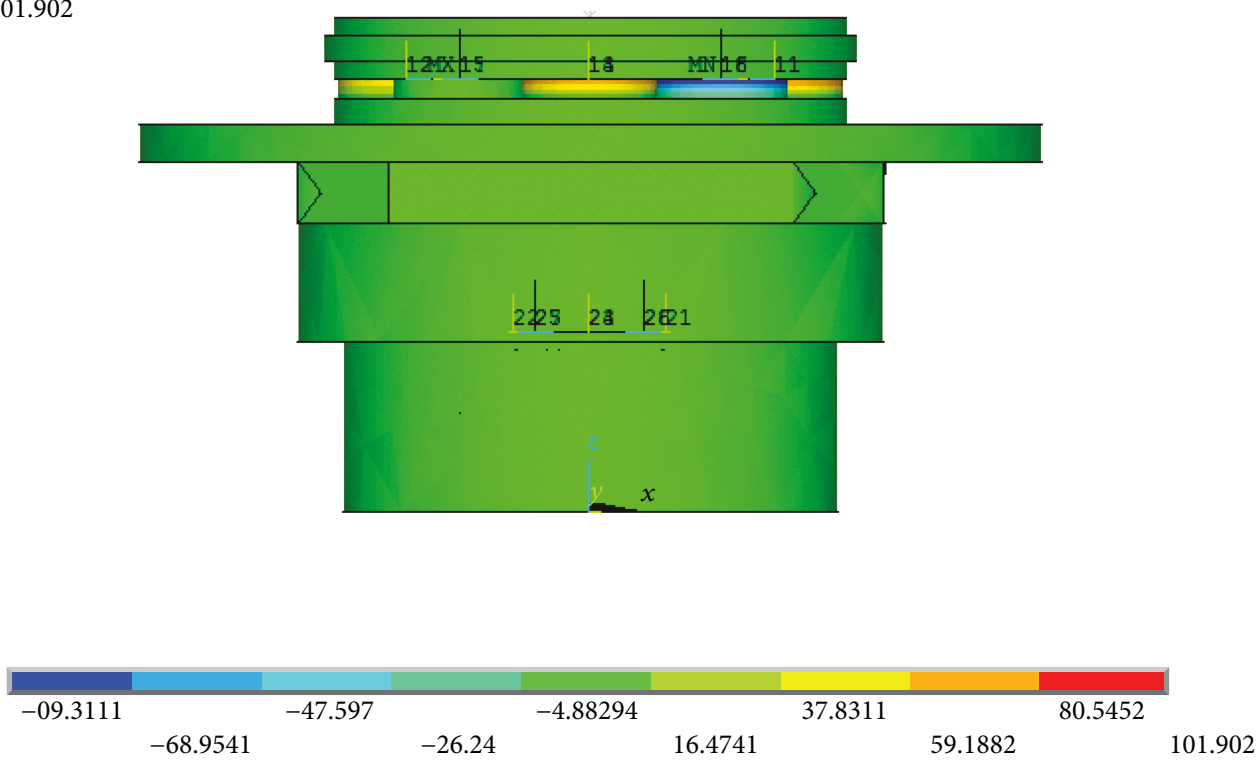

(a)

Nodal solution

ANSYS

STEP $=8$

SUB $=5$

TIME $=8$

VOLT (AVG)

$\mathrm{DMX}=0.230 E-06$

$\mathrm{SMN}=-90.3111$

SMX $=101.902$

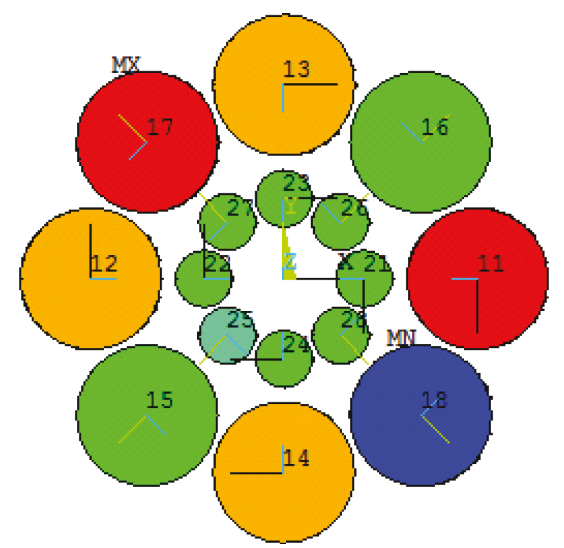

NOV 152016

17:06:28

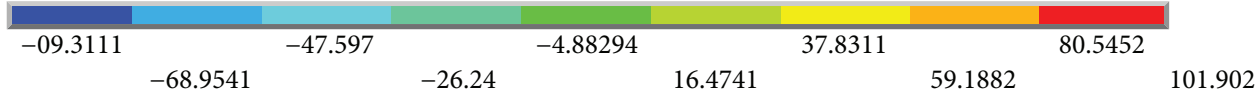

(b)

FigURE 10: Strain nephogram of 12-DOF force/acceleration sensor under combined loadings. 
TABLE 9: Third-time decoupling analysis results of the 6-axis acceleration sensing module under composite loads.

\begin{tabular}{lcccc}
\hline Load category & Input value & Output of first-time decoupling & Output of third-time decoupling & Relative error \\
\hline$A_{x}$ & $98 \mathrm{~m} / \mathrm{s}^{2}$ & $88.341 \mathrm{~m} / \mathrm{s}^{2}$ & $97.966 \mathrm{~m} / \mathrm{s}^{2}$ & $0.0350 \%$ \\
$A_{y}$ & $98 \mathrm{~m} / \mathrm{s}^{2}$ & $104.347 \mathrm{~m} / \mathrm{s}^{2}$ & $97.977 \mathrm{~m} / \mathrm{s}^{2}$ & $0.0232 \%$ \\
$A_{z}$ & $98 \mathrm{~m} / \mathrm{s}^{2}$ & $98.038 \mathrm{~m} / \mathrm{s}^{2}$ & $97.994 \mathrm{~m} / \mathrm{s}^{2}$ & $0.0064 \%$ \\
$\alpha_{x}$ & $1000 \mathrm{rad} / \mathrm{s}^{2}$ & $1093.901 \mathrm{rad} / \mathrm{s}^{2}$ & $999.337 \mathrm{rad} / \mathrm{s}^{2}$ & $0.0663 \%$ \\
$\alpha_{y}$ & $1000 \mathrm{rad} / \mathrm{s}^{2}$ & $1304.087 \mathrm{rad} / \mathrm{s}^{2}$ & $1001.338 \mathrm{rad} / \mathrm{s}^{2}$ & $-0.1338 \%$ \\
$\alpha_{z}$ & $1000 \mathrm{rad} / \mathrm{s}^{2}$ & $1001.562 \mathrm{rad} / \mathrm{s}^{2}$ & $999.991 \mathrm{rad} / \mathrm{s}^{2}$ & $0.0009 \%$ \\
\hline
\end{tabular}

TABLE 10: Third-time decoupling analysis results of the 6-axis force sensing module under composite loads.

\begin{tabular}{lcccc}
\hline $\begin{array}{l}\text { Load } \\
\text { category }\end{array}$ & $\begin{array}{l}\text { Input } \\
\text { value }\end{array}$ & Output of first-time decoupling & Output of third-time decoupling & Relative error \\
\hline$F_{x}$ & $100 \mathrm{~N}$ & $77.528 \mathrm{~N}$ & $100.296 \mathrm{~N}$ & $0.2962 \%$ \\
$F_{y}$ & $100 \mathrm{~N}$ & $92.630 \mathrm{~N}$ & $100.096 \mathrm{~N}$ & $0.0964 \%$ \\
$F_{z}$ & $100 \mathrm{~N}$ & $88.111 \mathrm{~N}$ & $99.728 \mathrm{~N}$ & $-0.2718 \%$ \\
$M_{x}$ & $10 \mathrm{~N} \cdot \mathrm{m}$ & $9.914 \mathrm{~N} \cdot \mathrm{m}$ & $10.006 \mathrm{~N} \cdot \mathrm{m}$ & $0.0585 \%$ \\
$M_{y}$ & $10 \mathrm{~N} \cdot \mathrm{m}$ & $10.091 \mathrm{~N} \cdot \mathrm{m}$ & $9.987 \mathrm{~N} \cdot \mathrm{m}$ & $-0.1258 \%$ \\
$M_{z}$ & $10 \mathrm{~N} \cdot \mathrm{m}$ & $9.936 \mathrm{~N} \cdot \mathrm{m}$ & $10.000 \mathrm{~N} \cdot \mathrm{m}$ & $0.0036 \%$ \\
\hline
\end{tabular}

method was investigated. Based on the results, the following conclusions were obtained:

(1) The structure and measurement principle of the piezoelectric 12-DOF force and acceleration sensor were accurate. The spatial 6-axis force/torque and 6-axis linear acceleration/angular acceleration were provided efficiently.

(2) With respect to the inertial field, the spatial structure of the piezoelectric 12-DOF force and acceleration sensor causing the generation of crossing couplings were between $a_{y}$ and $\alpha_{x}$, as well as between $a_{x}$ and $\alpha_{y}$, in the 6-DOF acceleration sensing part. The 6axis force sensing part and the crossing couplings were generated in the directions of $a_{x}, \alpha_{y}, F_{x}$, and $M_{y}$. With respect to all the directions of $a_{y}, \alpha_{x}, M_{x}$, and $F_{y}$ the directions of $a_{z}$ and $F_{z}$, in conjunction with the directions of $\alpha_{z}$ and $M_{z}$, were identical. However, the sensor's static crossing couplings were different to those of traditional nonlinear coupling and were eliminated by the decoupling method used in this study.

(3) The natural frequency of the 12-DOF force/acceleration sensor, in all directions, exceeded $11 \mathrm{kHz}$, and this produced a high natural frequency and a wide working frequency band. The sensitivity of the 12DOF force/acceleration sensor was slightly lower, although the natural frequency was relatively higher to those of other types of multiaxis accelerometers and force sensors.

(4) Based on the decoupling process presented in this study, the final decoupling results indicate that the relative error of the $12-\mathrm{DOF}$ force/acceleration sensor was less than $1 \%$.

\section{Conflicts of Interest}

The authors declare no conflict of interest.

\section{Acknowledgments}

This research is supported by the National Natural Science Foundation of China (no. 51475060) and the Natural Foundation Project of Chongqing China CSTC (no. CSTC2015JCYJBX0071).

\section{References}

[1] A. Winkler and J. Suchý, "Dynamic force/torque measurement using a 12 DOF sensor," in 2007 IEEE/RSJ International Conference on Intelligent Robots and Systems, pp. 18701875, San Diego, CA, USA, November 2007.

[2] J. G. García, A. Robertsson, J. G. Ortega, and R. Johansson, "Force and acceleration sensor fusion for compliant robot motion control," in Proceedings of the 2005 IEEE International Conference on Robotics and Automation, pp. 2709-2714, Barcelona, Spain, April 2005.

[3] J. Liu, M. Li, L. Qin, and J. Liu, "Principle research on a single mass piezoelectric six-degrees-of-freedom accelerometer," Sensors, vol. 13, no. 8, pp. 10844-10855, 2013.

[4] W. G. Zhang, "A new distributing and decoupling method of six-degree-of-freedom force sensors," Journal of Nanjing University of Aeronautics \& Astronautics, vol. 31, no. 2, pp. 221-222, 1999.

[5] L. P. Chao and C. Y. Yin, "The six-component force sensor for measuring the loading of the feet in locomotion," Materials \& Design, vol. 20, no. 5, pp. 237-244, 1999. 
[6] JR3 Inc., "12 DOF sensors with acceleration compensation," http://jr3.com/products/12dof_overview.html.

[7] F. Gao, Z. L. Jin, X. C. Zhao, and J. L. Li, "12-DOF Force and Acceleration Wrist Sensor of Robot," China Patent 02122567.2, 2002.

[8] F. L. Ni et al., "A 12-DOF Sensor in the End of Robot and its Design Method," China Patent 201510696343.0, 2015.

[9] K. Li, Research on 12 - DOF Sensor and Application for the End of Robot Arm, [M.S. thesis], Dept. Mechanic. Eng., Harbin Institute of Technology, Harbin, China, 2016.

[10] T. Kröger, D. Kubus, and F. M. Wahl, "12D force and acceleration sensing: a helpful experience report on sensor characteristics," in 2008. ICRA 2008. IEEE International Conference on Robotics and Automation, pp. 3455-3462, Pasadena, CA, USA, May 2008.

[11] J. Liu et al., "A piezoelectric 12-DOF Sensor," China Patent 200810070130.7, 2008.

[12] L. Qin, C. Jiang, J. Liu, and Y. Duan, "Design and calibration of a novel piezoelectric six-axis force/torque sensor," in Proceedings Volume 8321, Seventh International Symposium on Precision Engineering Measurements and Instrumentation, pp. 83210G1-83210G9, Lijiang, China, November 2011.

[13] H. Y. Lv, L. Qin, and J. Liu, "Principle research on a single mass six-degree-of-freedom accelerometer with six groups of piezoelectric sensing elements," IEEE Sensors Journal, vol. 15, no. 6, pp. 3301-3310, 2015. 


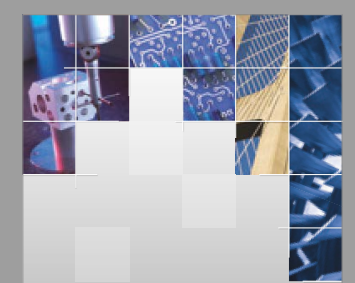

\section{Enfincering}
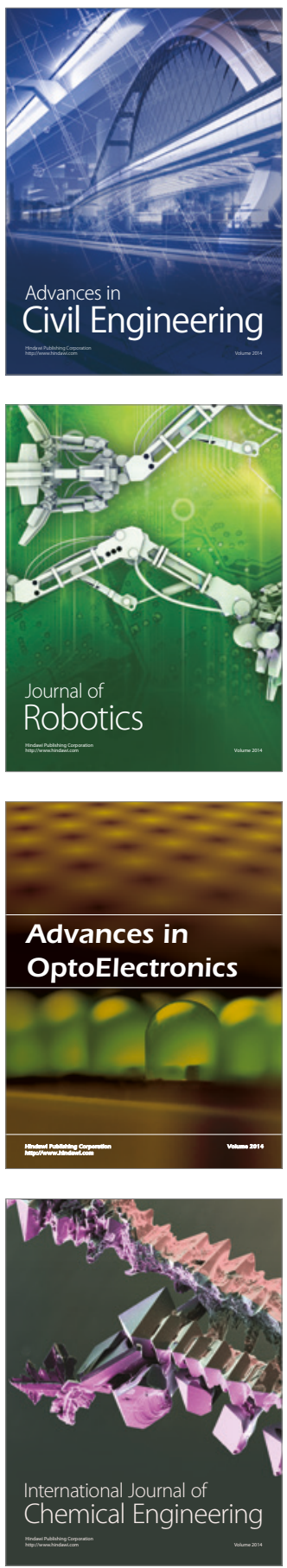

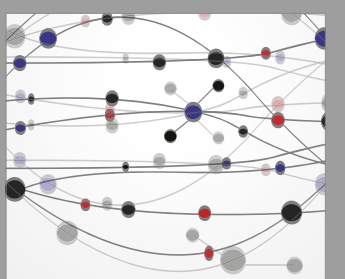

The Scientific World Journal

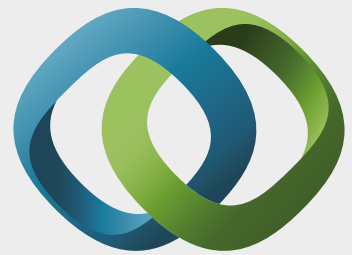

\section{Hindawi}

Submit your manuscripts at

https://www.hindawi.com
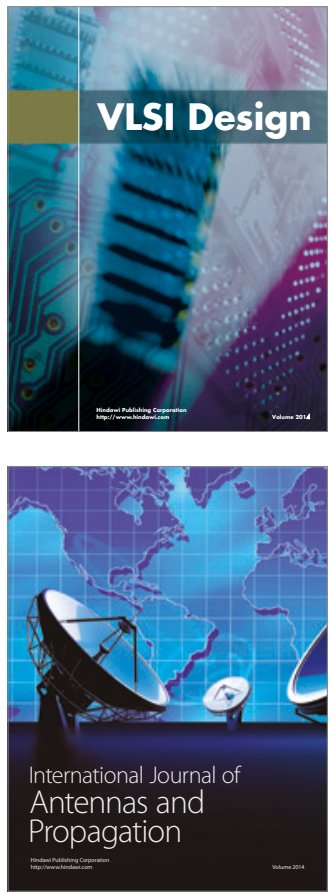

\section{Rotating}

Machinery
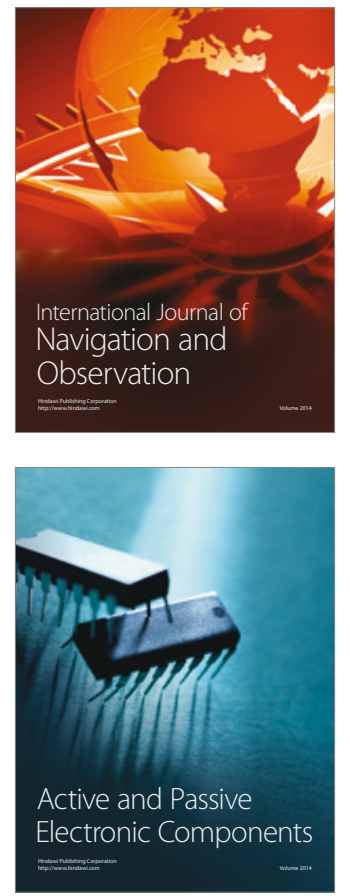
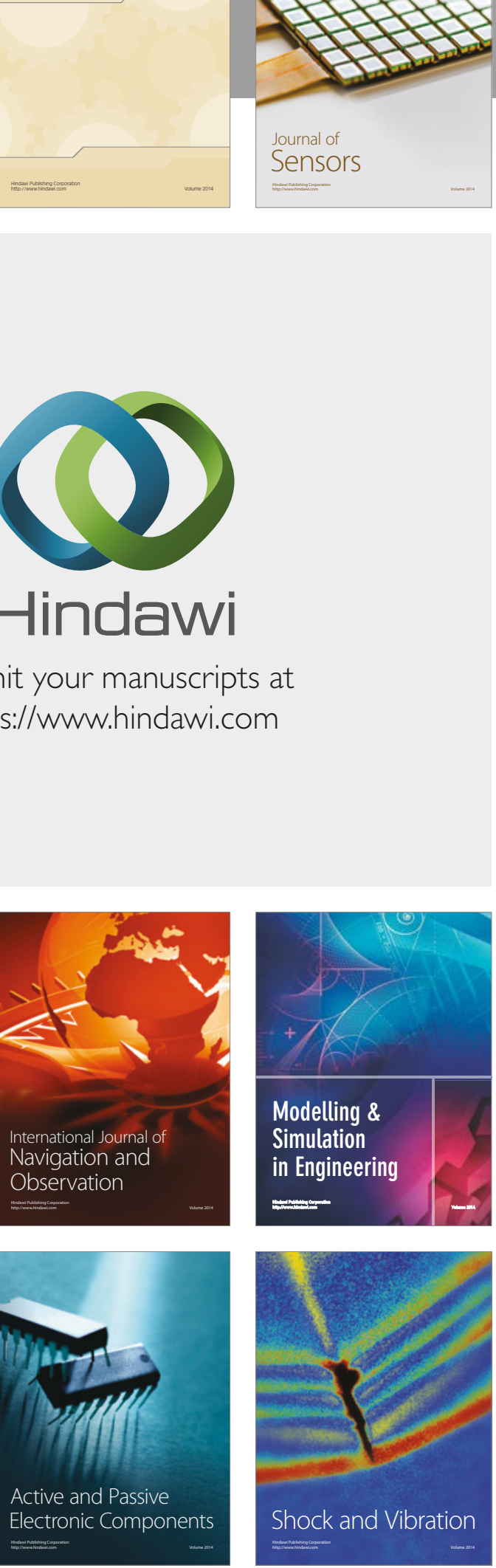
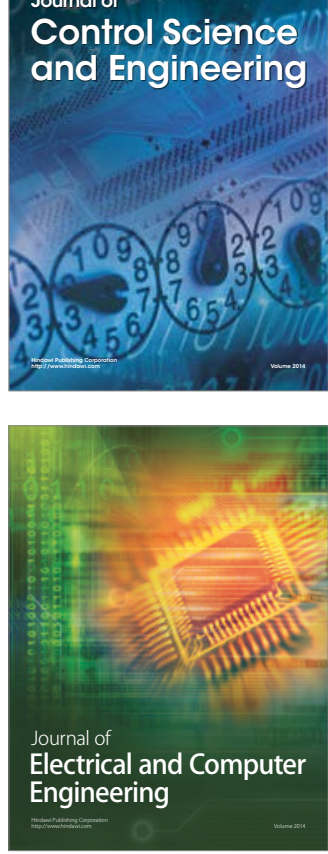

Distributed

Journal of

Control Science

and Engineering
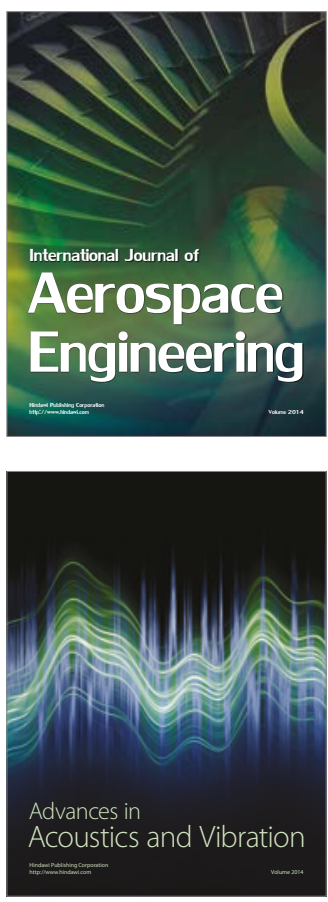

Sensor Networks 